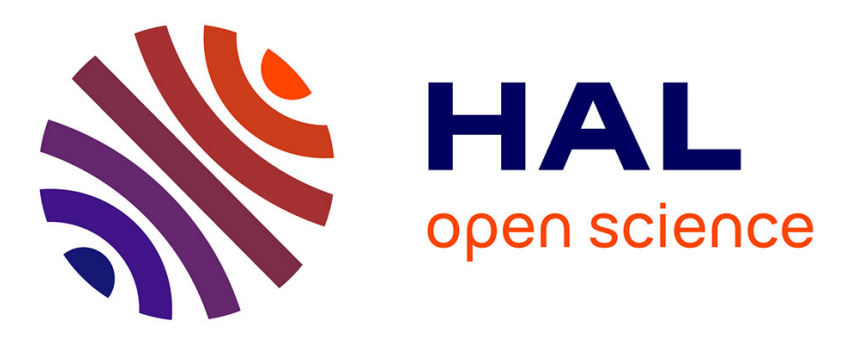

\title{
Dynamic Effects in Voltage Pulsed Atom Probe
}

Loïc Rousseau, Antoine Normand, Felipe Morgado, Leigh Stephenson, Baptiste Gault, Kambiz Tehrani, François Vurpillot

\section{To cite this version:}

Loïc Rousseau, Antoine Normand, Felipe Morgado, Leigh Stephenson, Baptiste Gault, et al.. Dynamic Effects in Voltage Pulsed Atom Probe. Microscopy and Microanalysis, 2020, 26 (6), pp.1133-1146. 10.1017/S1431927620024587 . hal-03013684

\section{HAL Id: hal-03013684 https://hal.science/hal-03013684}

Submitted on 3 May 2021

HAL is a multi-disciplinary open access archive for the deposit and dissemination of scientific research documents, whether they are published or not. The documents may come from teaching and research institutions in France or abroad, or from public or private research centers.
L'archive ouverte pluridisciplinaire $\mathbf{H A L}$, est destinée au dépôt et à la diffusion de documents scientifiques de niveau recherche, publiés ou non, émanant des établissements d'enseignement et de recherche français ou étrangers, des laboratoires publics ou privés. 


\title{
Dynamic Effects in Voltage Pulsed Atom Probe
}

\author{
Loïc Rousseau ${ }^{1,2 \star}$ (D), Antoine Normand ${ }^{1}$, Felipe F. Morgado ${ }^{3}$, Leigh Stephenson ${ }^{3}$, Baptiste Gault ${ }^{3,4}$ (D), Kambiz Tehrani ${ }^{2}$ \\ and François Vurpillot ${ }^{1}$ \\ ${ }^{1}$ Groupe Physique des Matériaux, Université de Rouen, Saint Etienne du Rouvray, Normandie 76800, France; ${ }^{2}$ ESIGELEC, Avenue Galilée, Saint Etienne du Rouvray, \\ Normandie 76800, France; ${ }^{3}$ Max-Planck Institut für Eisenforschung GmbH, Düsseldorf D-40237, Germany and ${ }^{4}$ Department of Material, Royal School of Mines, \\ Imperial College, London, UK
}

\begin{abstract}
Atom probe tomography (APT) is particularly suited for the analysis of nanoscale microstructural features in metallic alloys. APT has become important in the quantitative assessment at high spatial resolution of light elements, which are notoriously difficult to analyze by electron- or X-ray-based techniques. These control the physical properties of high-strength materials and semiconductors. However, the mass spectrometer of state-of-the-art commercial atom probes with the highest spatial precision and detection efficiency are optimized for elements with mass-to-charge ratios corresponding to Fe and neighboring elements. Little is known on the theoretical performances for light elements. Here, we discuss the theoretical instrumental performance of one such instrument using accurate three-dimensional transient electrostatic simulations in a time-varying field approach. We compare the simulations to experimental measurements obtained on an FeBSi bulk-metallic glass. Dynamics effects during the ion's flight are revealed when examining multi-hit mass-to-charge correlations, and we demonstrate their influence on the mass resolution. The model reveals significant differences in ion projection as a function of the mass. We discuss how these chromatic aberrations affect the spatial precision. This approach shows that by tuning the shape of the voltage pulses used to trigger field evaporation, minimizing the influence of these detrimental dynamic effects is possible.
\end{abstract}

Key words: atom probe, chromatic aberrations, dynamic effects, Saxey's approach, time-of-flight mass spectrometry

\section{Introduction}

Atom probe tomography (APT) is increasingly recognized as a critical technique to investigate materials at the near-atomic scale, providing complementary information to, for example, transmission electron microscopy. APT allows to map the threedimensional (3D) distribution of the different atoms in a probed volume of a given material. This instrument is particularly suited for the nanoscale analysis of low mass atoms, diluted or segregated, and is a key instrument in the study of hydrogen embrittlement for instance (Chen et al., 2017; Takahashi et al., 2019; Breen et al., 2020). The precise analysis of hydrogen, boron, carbon, nitrogen, and other light elements has become crucial to the design future materials but also to predict the in-service behavior of existing materials and potentially develop strategies to enhance their lifetimes. The main strength of APT compared with other nano-analytical techniques is its intrinsic ability to provide the tomographic reconstruction of the distribution of elemental species in the analyzed volume with high sensitivity and accuracy. This ability is combined with a sub-nanometer spatial precision capability (De Geuser \& Gault, 2020). In APT, an intense evaporation field in the range of $10-60 \mathrm{~V} / \mathrm{nm}$ generated at the apex of a needle-shaped specimen, provokes the removal of surface atoms in the form of ions. It is produced by the application of a strong positive voltage $V$ between the specimen and a counter electrode (CE). The sharp specimen has a near-hemispherical tip, with a radius $R$ in the range of $50-100 \mathrm{~nm}$. The pointed nature of the specimen enhances greatly the surface field evaporation $F$ so that a few kilovolts are generally sufficient to reach the required critical field $\left[F_{\mathrm{EV}}=\left(V / k_{\mathrm{f}} R\right)\right.$, with $k_{\mathrm{f}}$ a constant in the range of (2-8), which depends on the specimen shape and the system geometry] (CAMECA LEAP 5000 Atom Probe, 2020; EIKOS, 2020; Kelly et al., 1996; Bémont et al., 2003; Deconihout et al., 2003). Ion emission can be triggered by short positive voltage pulses applied to the specimen or negative voltage pulses applied to the CE (Larson et al., 1996), which enables elemental identification by means of time-of-flight mass spectrometry (TOF-MS). Atom probes with the highest chemical sensitivity for low mass atoms and with the best spatial precision are straight flight path atom probes equipped with the latest generation of positionsensitive detectors. Equipped with funnel-shaped micro-channel plates on the entry-face of the detector for conversion and amplification, the detection efficiency reaches $80 \%$ and is constant over a very wide range of atomic masses. In straight flight path atom probes, i.e., not fitted with an energy compensation device such as a reflectron, the ions are directly collected on the bare surface of the detector placed a few centimeters in front of the sample. The best spatial performances are then obtained under low base temperature, using voltage pulses to trigger field evaporation (Gault et al., 2010a). Laser-assisted field evaporation must be 
avoided to reduce image blurring induced by detrimental thermal effects (Cerezo et al., 2006; Gault et al., 2010b; Gruber et al., 2011). Yet, using voltage pulsed mode on a straight flight path AP, the mass resolution and associated elemental sensitivity remain key limitations. Indeed, the mass resolution must be sufficient to distinguish peaks pertaining to elements of close mass-to-charge ratios, and for small peaks to stand above the background. The ability to quantify these elements is related to the intrinsic mass resolving power of the instrument.

The mass resolution is often quoted at half maximum, typically $M / \delta M>1,000$, but APT's analytical performances are critically dependent on the width of mass peak at 10 or $1 \%$ of the maximum (Larson, 2013). In voltage pulsed atom probe (VP-AP), field evaporated ions leave the sample at slightly different times relative to the pulse maximum (start-time) because of the stochastic nature of the evaporation process (Müller, 1951). This causes some ions of the same nature to acquire slightly smaller kinetic energies, and therefore slightly slower velocities compared with the peak maximum, resulting in a higher mass tail in the mass spectrum. In order to improve the mass resolution of the instrument, it is mandatory first, to control the quality of the voltage pulse (VP) applied to the sample, and second to accurately define the design of the micro-electrode on which the VP is applied. These optimizations were done in commercial instrument using the concept of the CE, proposed by Kelly et al. (1996). The geometry of the instrument was designed to minimize energy errors for ions of medium mass (around $30 \mathrm{amu}$ ). Mass resolution achieving $500 \mathrm{FWHM}$ in the analysis of $\mathrm{Al}^{+}(\mathrm{m} / n=$ 27) or $\mathrm{Fe}^{2+}(m / n=28)$ was experimentally demonstrated (Larson et al., 1996). The conditions for the best mass resolution performance are relatively well-known for this range of mass and at the center of the detector. However, out of this range, the performances are less well documented at least from a theoretical perspective. Indeed, the performance of the CE was established more than 20 years ago on simplified design of the instrument (Larson et al., 1996). In particular, the impact of a realistic VP shape, for ions of low mass, and out of the central $z$-axis need to be evaluated and optimized. The most recent study on the influence of the VP on ion trajectory (Zhao et al., 2015) demonstrates optimal pulse shapes, but the model was also limited to a simplified design and to a unique type of ion ( $\mathrm{Al}$ atoms). A high resolution without energy compensation is possible with a subnanosecond VP with a flat top. Such a pulse shape should strongly minimize energy deficits. However, experimentally, this pulse is difficult to control, and the mass resolution is not as high as expected due to ripples on the pulse shape. The calculation of theoretical mass spectra for ions of low mass, in a realistic description of the ultra-high vacuum chamber, is mandatory to push further the optimization of the instrument. It is also mandatory to evaluate the influence of the angles of ion emission. A study of Loi et al. (2013) gives the method to accurately estimate the influence of the exact geometry of the system on the ion projection. In particular, the dependence of the field factor $k_{\mathrm{f}}$ and the compression factor $\xi$ was calculated in this work in the exact geometry of the local electrode atom probe (LEAP). Through a detailed electrostatic modeling of an LEAP, they made different simulations by varying various parameters relating to the microscope and specimen geometry. This demonstrates that reconstruction parameters are not static through a standard analysis. Here, we adapted this electrostatic model to a state-of-the-art microscope, but using a time-varying approach, which enables the study of the spatiotemporal effects in the projection of ions. We demonstrate that the mass resolution is not only dependent of the VP shape but also of the ion mass and the exact geometric configuration. This encouraged us to investigate the dynamic effect due to the pulse to find the best compromise to optimize the performance of APT analysis. These numerical results are compared with experimental measurements made on an FeBSi alloy on a straight flight path LEAP in VP mode (LEAP 5000XS). FeBSi alloy allows us to study a wide range of mass-to-charge from $\mathrm{H}^{+}(1 \mathrm{amu}), \mathrm{B}^{2+}$ (main peak at $5.5 \mathrm{amu}$ ), $\mathrm{B}^{+}$(main peak at $11 \mathrm{amu}$ ), $\mathrm{Si}^{2+}$ (main peak at $14 \mathrm{amu}$ ) to $\mathrm{Fe}^{2+}$ (main peak at $28 \mathrm{amu}$ ). Dynamic effects are evidenced by means plotting a correlation map of multi-hits event as proposed by Saxey (2011). We have also compared mass resolutions with different pulse configurations (VP on sample or on the CE). Solutions are discussed to efficiently improve performances.

\section{Material and Methods}

\section{Atom Probe Basics}

Atom probe instruments (Fig. 1) are designed to field-evaporate atoms from a sharp needle-shaped specimen either by voltage pulses transmitted by a CE or thermally enhanced by laser pulses. The geometry of the instrument modeled in this paper (an LEAP $5000 S$ ) is defined by a complex assembly of electrodes, enabling first a correct transmission of the VP to the CE placed in front of the sample, and second to ensure a defined projection of ions toward the detector. TOF-MS is possible through the measurement of ions TOF from the precise time of application of the VP on the $\mathrm{CE}$ to the time of detection of ions onto the detector.

The output data stream is indeed a series of coordinates giving the location and time of impact at a position-sensitive detector, from which we can deduce chemical identities by TOF-MS and original positions using a suitable reverse projection algorithm. An ion leaving the surface tends to deflect toward the detector. In the first steps of flight, ions are emitted with an angle $\theta$ at the surface of the hemispherical cap. Due to the electrostatic influence of the needle shape geometry and surrounding electrodes, the angle is compressed in a first approach into a projected angle $\theta^{\prime}$. The ratio $\theta / \theta^{\prime}$ defines the image compression that is required to reconstruct image from APT data.

In this paper, dynamic mass spectrometry effects will be estimated in VP mode, which means, both deviations in time (influencing mass estimation) and space (influencing image reconstruction) compared with the static model will be evaluated. In an LEAP, the tip is held at a voltage $V_{\mathrm{DC}}$, and a VP of absolute amplitude equal to $\alpha_{\mathrm{p}} V_{\mathrm{DC}}$ triggers the field evaporation of atoms. TheTOF $t_{\mathrm{f}}$ can be measured only if the evaporation is triggered at $t_{0}$ by the VP applied on the CE. It is assumed that ions have no initial velocity, and an instantaneous acceleration to the final speed. Following Figure 1, the flight length is considered as the distance $L$ from the sample to the detector (the acceleration distance from the tip to the $\mathrm{CE}$ is neglected). When ions arrived on the detector, their whole energy is converted into kinetic energy. The mass over charge ratio $M(\mathrm{amu})$ is written as (Pareige et al., 2016)

$$
\frac{m}{n}=2 e \frac{V_{\mathrm{DC}}\left(1+\alpha_{\mathrm{p}}\right)}{L^{2}}\left(t_{\mathrm{f}}-t_{0}\right)^{2}
$$

where $m$ is the mass of the ion, $n$ the charge, $e$ the electronic charge, $V_{\mathrm{DC}}(\mathrm{kV})$ the continuous high voltage, $\alpha_{\mathrm{p}}$ the pulse contribution, $L(\mathrm{~m})$ the distance sample to the detector, $t_{\mathrm{f}}(\mu \mathrm{s})$ the event 


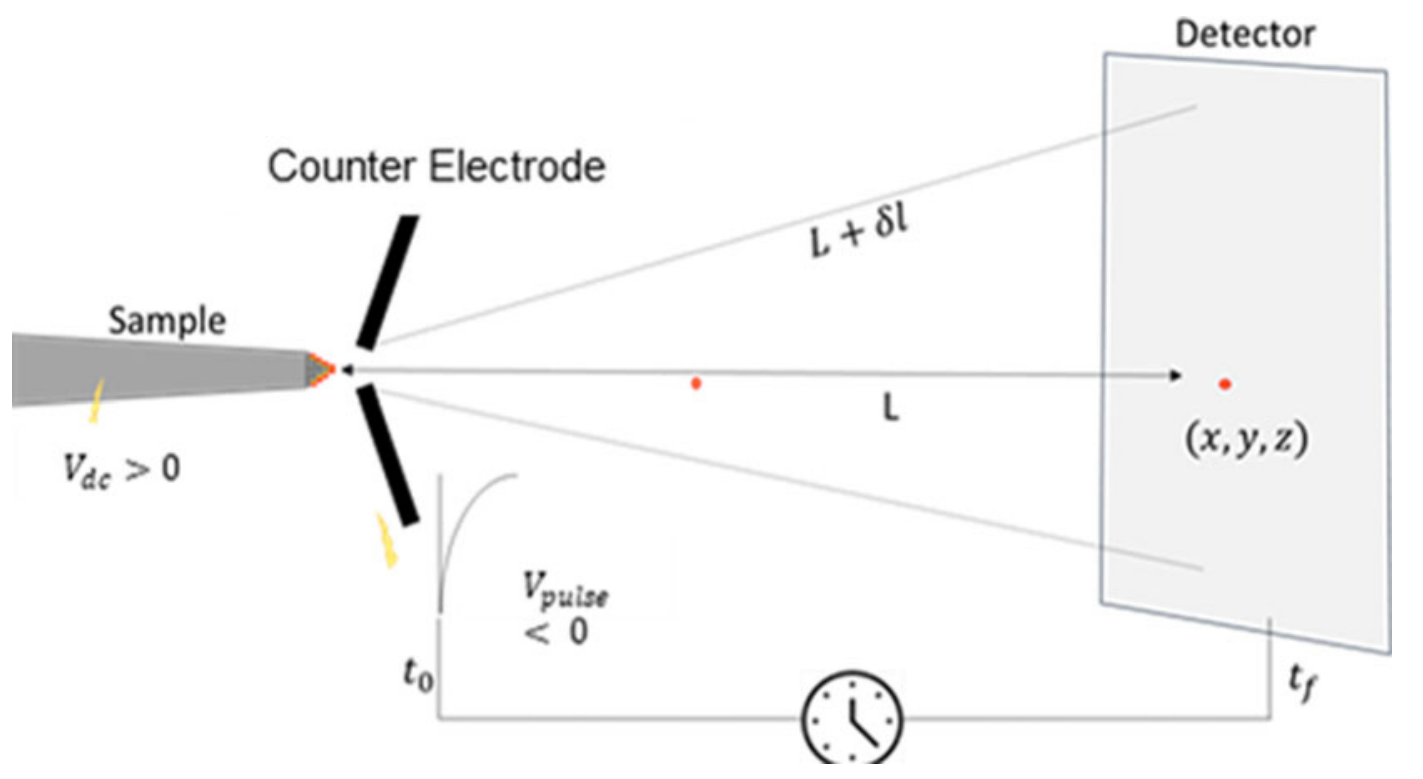

Fig. 1. Schematic image illustrating the physical geometry of a tip in front of a CE. A high voltage $V_{D C}$ is applied on the sample to generate a high electric field; the $\mathrm{CE}$ is biased to a negative pulse voltage from several $\mathrm{kV}$. Time-of-flight spectrometry principle is used to calculate $\mathrm{m} / \mathrm{n}$ ratio on each evaporated ion detected on the detector placed at a distance $L$ from the tip.

time on the detector, and $t_{0}$ the time triggered on the maximum of the pulse. We may note that negative voltage pulses are experimentally applied to the CE placed a few micrometres in front of the sample $(10-100 \mu \mathrm{m})$. It allows the efficient propagation of high $\mathrm{VP}$ at a high repetition rate. The high field evaporation is achieved by the voltage difference between the tip and the CE. This high VP of nanosecond duration creates a high evaporation field $(F)$ on the surface of the tip apex (Vurpillot, 2016):

$$
F=\frac{V}{k_{\mathrm{f}} R}
$$

where $V \sim V_{\mathrm{DC}}+V_{\mathrm{p}}, k_{\mathrm{f}}$ the field evaporation factor, and $R$ the apex radius and $V_{\mathrm{p}}$ the absolute amplitude of the VP. Because $V_{\mathrm{p}}$ is not directly applied on the sample, the additional surface field produced is not exactly equal to $V_{\mathrm{p}} / k_{\mathrm{f}} R$. However, the coupling factor is close to $100 \%$ in an LEAP configuration with a small tip to CE distance. The ionization process of the material follows a probabilistic law (an Arrhenius law) that is commonly described by:

$$
K\left(s^{-1}\right) \propto \exp \left(a \alpha_{\mathrm{p}}(1-f)\right)=\exp \left(-\frac{C}{k_{\mathrm{B}} T} \alpha_{\mathrm{p}}\left(1-\frac{F}{F_{\mathrm{ev}}}\right)\right)
$$

where $F_{\mathrm{ev}}$ is the evaporation field constant of the material, $T$ the temperature, and $C$ the asymptotic zero field energy of the evaporation barrier. The parameter " $a$ " is a key constant in this equation $\left(a=C / k_{\mathrm{B}} T\right)$, and $f$ is the ratio between field and evaporation field. In this paper, the VP is modeled as a double exponential shape to describe the asymmetric nature of the pulse:

$$
V_{\mathrm{p}}=U\left(\exp ^{V t}-\exp ^{W t}\right)
$$

where $U, V, W$ are constant values. The constant $C$ is documented in Miller \& Forbes (2014) and is at maximum about 2-3 eV for refractory metals. We will consider for the sake of simplicity a value of $C \sim 1 \mathrm{eV}$. Temperature is generally fixed in AP between 40 and $100 \mathrm{~K}$, so that $a \sim$ [100-400] (Fig. 2).

By combining the evaporation rate and the VP, the temporal probability of evaporation follows a very narrow peak centered on the VP maximum of a width that corresponds to the top of the pulse ( $10 \%$ maximum of the top of pulse), where the pulse can be described accurately with a parabolic shape. It is, therefore, possible to approximate the evaporation pulse by a Gaussian distribution. Using the same approximation of Zhao et al. (2015) and considering $\delta=1-f$, the relative shift to the maximum field applied to the tip. The approximate theoretical distribution of ions being evaporated with a shift $\delta$ to the maximum field can be written as follows:

$$
D_{\delta}^{\mathrm{F}}=\mathrm{e}^{-a \delta}\left(\sqrt{\frac{a}{\pi \delta}}\right)
$$

Assuming equations (1) and (2), $D_{\delta}^{\mathrm{F}}$ is also assumed to be directly linked to the relative energy deficit density distribution $D_{\delta}^{\mathrm{V}}$. This equation has a singular value for $\delta=0$ and an asymptotic exponential tail for significant high deficit. In the log scale, the tail of this equation presents a slope proportional to the constant $a$. A mass spectrum is calculated using equation (1), by assuming all ions are evaporated at the top of the pulse, and with the maximum kinetic energy. During the experimentation, it is considered that the majority of the ions are evaporated at the maximum of the pulse during a period $\delta t$ with a small voltage deficit $\delta V$ (or energy deficit $\delta E=\delta V / V$ ). Following the theoretical results, this is obviously not true. Errors induced by the pulsed nature of the experimental process degrade the mass resolution. To optimize the mass spectrometer performance of the instrument, it is mandatory to evaluate all kinds of errors. The theoretical energy resolution can be further degraded by electronic precision of the instrumentation $(\delta t / t)$, and errors in flight length estimation $(\delta L / L)$ and the mass resolution should be estimated by 


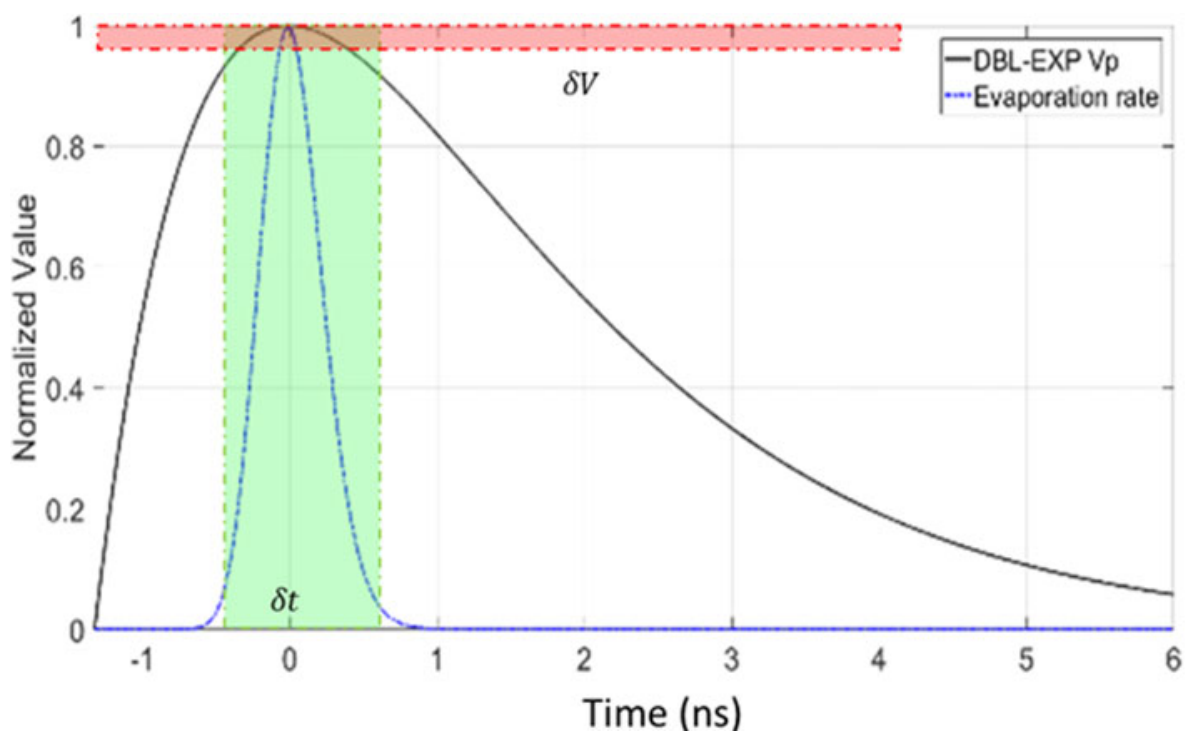

Fig. 2. Contributions to mass resolution degradation in voltage pulsed atom probe: Departure time $\delta t$ (green interval) and dispersion in potential energy $\delta E=n e \delta V$ (red interval). The black curve is a double exponential VP of $T_{\text {width }}=2 \mathrm{~ns}$, and the dashed blue curve is the corresponding probability of ionization calculated with the Arrhenius law [equation (3)].

the common quadratic sum of errors:

$$
\frac{M}{\delta M}>\frac{1}{\sqrt{\left(\sum\left(\delta V^{2} / V^{2}\right)\right)+\left(2 \sum\left(\delta t^{2} / t^{2}\right)\right)+\left(\sum\left(\delta L^{2} / L^{2}\right)\right)}}
$$

Dynamic effects induce additional mass spectrometry errors in APT during the flight of the ions. As it will be simulated, the relationship between $D_{\delta}^{\mathrm{V}}$ and $D_{\delta}^{\mathrm{F}}$ is true if an ion can acquire the total potential energy brought by the application of the VP. In reality, the voltage distribution around the tip evolves strongly during the ion flight, so that some ions could get more or less energy compared with a static calculation. These effects must be evaluated to optimize the instrument.

\section{Experimental Setup and Data Treatment}

The material of interest in this study is a metallic sample FeBSi. This material was chosen since several range of ion masses can be studied simultaneously in a single material $\mathrm{H}^{+}, \mathrm{B}^{2+}, \mathrm{B}^{+}, \mathrm{Si}^{2+}$, and $\mathrm{Fe}^{2+}$. To field evaporate the material, the specimen is first prepared as a sharply pointed needle with a hemispherical apex of $50 \mathrm{~nm}$ placed at about $10 \mathrm{~cm}$ in front of an ion detector. A CE is added in front of the sample at $40 \mu \mathrm{m}$ (Fig. 3). The electric evaporation field is produced from a high positive DC voltage ( few $\mathrm{kV}$ ) applied on the sample and a high negative pulse voltage corresponding to a fraction of the required voltage (between 10 and $25 \%$ of $\left.V_{\text {total }}\right)$. Analyses were performed at $40 \mathrm{~K}$. Experimental mass spectra were optimized using the Cameca software IVAS, using the conventional bowl correction method minimizing the systematic errors in applied voltage and flight length as a function of impact positions. Mass spectra were calculated using a $\mathrm{m} / \mathrm{n}$ bin resolution of $5 \times 10^{-3}$ Da without background noise corrections. Mass resolution was calculated at half maximum of each peak (FWHM), 10\% of the peak maximum (FWTM), and $1 \%$ of the peak maximum (FW1\%M). The FeBSi alloy is an amorphous metallic alloy, with high percentage of boron, randomly distributed, which is known to have a high evaporation field $(>50 \mathrm{~V} / \mathrm{nm})$ inside the Fe matrix $(F \sim 35 \mathrm{~V} / \mathrm{nm})$. As a result, conversely to pure materials, the evaporation of a boron atom is often followed by bursts of simultaneous evaporation of neighbor atoms. This phenomenon was described in the paper of De Geuser et al. (2007). As a result, the VP mode analysis of this material gives rise to a high occurrence of multi-impacts on the detector that are mainly due to instantaneous evaporation of atoms. To dissociate and visualize the impact of time errors and energy errors, we have visualized the correlation map proposed by Saxey (2011). In this analysis, multi-hits events, pair correlations are displayed across the entire mass spectrum. Usually, this method is used for laser AP experimentation to show the thermal effect of the laser pulse and mass/time dispersion. In this method, a histogram is calculated for pair of atoms (A, B) that are evaporated on the pulses (multi-hit events). The number of pairs for each two-dimensional (2D) coordinates of masses is calculated and represented by a color scale or gray scale. As compared with mass spectra, mass tails of different elements are dissociated in this diagram. In Saxey's diagram, the majority of the histogram counts are located at coincidences between major peaks in the mass spectrum. However, the most obvious features of the histogram are the lines that emanate from these coincident points. Correlated field evaporation gives rise to trails starting from theoretical couples $\left(m_{1}, m_{2}\right)$ of existing masses in the sample, moving from lower-left to upper-right. These trails are a signature of the simultaneous field evaporation of the two atoms constituting the pair. In covalent or ionic materials, this correlation can point out the presence of a dissociation of a molecule into two fragments and received on the detector. Correlation maps are a suitable method to visualize and deconvolute time and energy errors. Indeed, assuming that a couple of atoms of masses $\left(M_{1}=m_{1} / n_{1}\right.$, $\left.M_{2}=m_{2} / n_{2}\right)$ are simultaneously evaporated from the tip, these two atoms give rise to two impacts on the detector that are measured with masses $\left(M_{1}^{D}, M_{2}^{D}\right)$. We may understand the correlation in mass in experimental Saxey analysis in the case of a systematic voltage shift $(-\delta V)$, or in the case of a systematic temporal shift $(+\delta t)$. In the first case, and using equation (1), we can write the 


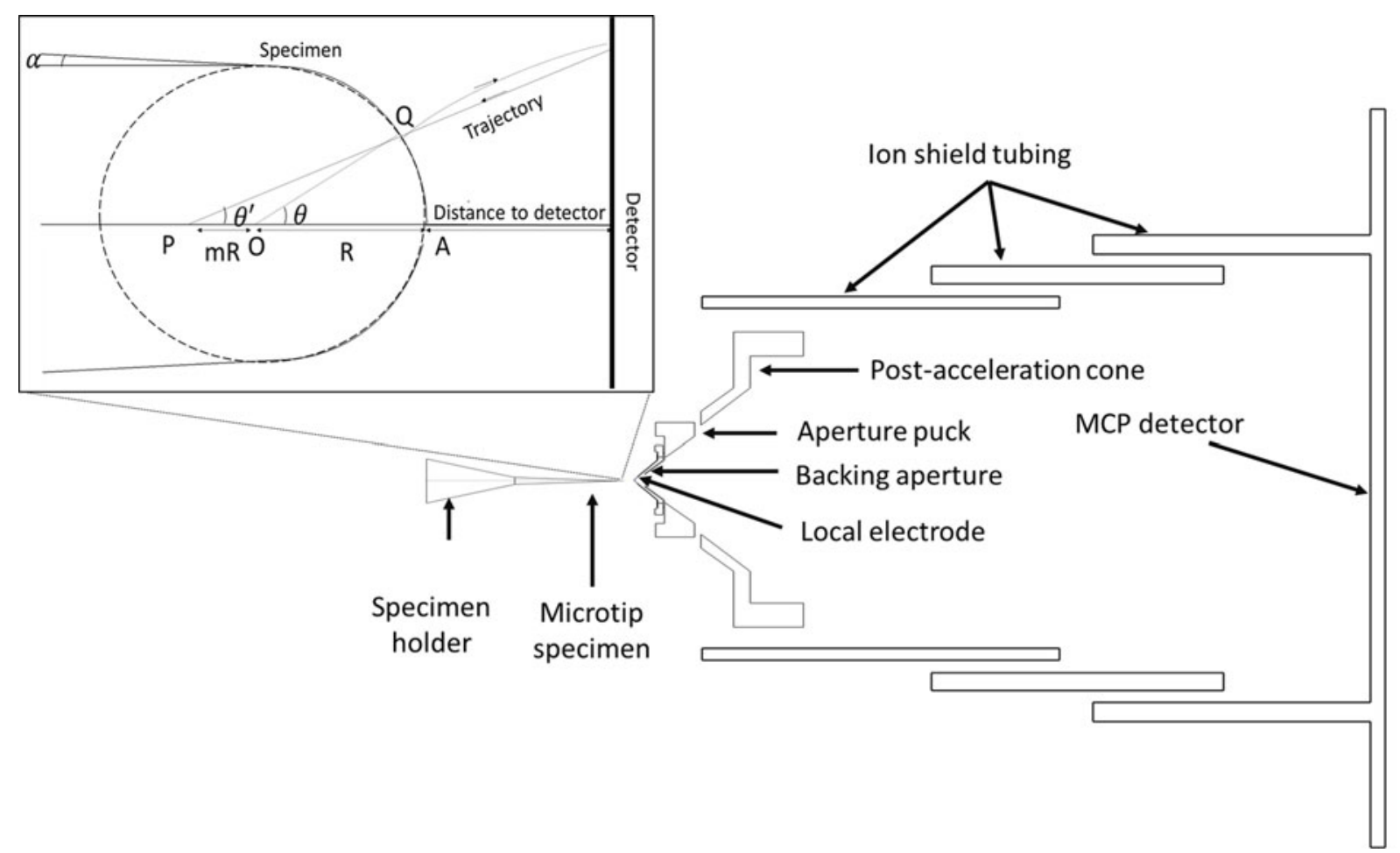

Fig. 3. Geometrical modeling of an LEAP 5000 on Lorentz-2E and description of the geometry of ion revers projection (not to scale).

measured masses as a function of:

$$
\left\{\begin{array}{l}
M_{1}^{D}=2 \mathrm{e} \frac{\boldsymbol{V}}{L^{2}}\left(t_{1}^{D}\right)^{2} \\
M_{2}^{D}=2 \mathrm{e} \frac{\boldsymbol{V}}{L^{2}}\left(t_{2}^{D}\right)^{2}
\end{array}\right.
$$

$t_{1}^{D}$ and $t_{2}^{D}$ are the measured times of flight, that are equal to $\left(t_{1}^{D}\right)^{2}=M_{1}\left(\boldsymbol{L}^{2} / 2 \mathrm{e}(V-\delta V)\right)$ and $\left(t_{2}^{D}\right)^{2}=M_{2}\left(\boldsymbol{L}^{2} / 2 \mathrm{e}(V-\delta V)\right)$, so that we can write, if $\delta V / V$ is small:

$$
\left\{\begin{array}{l}
M_{1}^{D}=M_{1} \times\left(1+\frac{\delta V}{V}\right) \\
M_{2}^{D}=M_{2} \times\left(1+\frac{\delta V}{V}\right)
\end{array}\right.
$$

The reduced masses $\left(\mu_{1}=M_{1}^{D} / M_{1}\right)$ and $\left(\mu_{2}=M_{2}^{D} / M_{2}\right)$ are therefore identical, and plotting $\mu_{1}$ as a function of $\mu_{2}$ gives a linear equation of slope equal to 1 .

In the second case, and using equation (1), we can write the measured masses as a function of $\delta t: M_{1}^{D}=2 \mathrm{e}\left(V / L^{2}\right)\left(t_{1}^{D}\right)^{2}$ : and $M_{2}^{D}=2 e\left(\boldsymbol{V} / L^{2}\right)\left(t_{2}^{D}\right)^{2}, t_{1}^{D}$ and $t_{2}^{D}$ are the measured times of flight that are equal to $t_{1}^{D}=t_{1}+\delta t=\left(M_{1}\left(L^{2} / 2 \mathrm{e} V\right)\right)^{1 / 2}+\delta t$ and $t_{2}^{D}=t_{2}+\delta t=\left(M_{2}\left(L^{2} / 2 \mathrm{e} V\right)\right)^{1 / 2}+\delta t$, so that we can write, if $\delta t / t$ is small:

$$
\left\{\begin{array}{l}
M_{1}^{D}=M_{1} \times\left(1+2 \frac{\delta t}{t_{1}}\right)=M_{1} \times\left(1+2 \times\left(\frac{2 \mathrm{eV}}{L^{2}}\right)^{1 / 2} \frac{\delta t}{M_{1}^{1 / 2}}\right) \\
M_{2}^{D}=M_{2} \times\left(1+2 \frac{\delta t}{t_{2}}\right)=M_{2} \times\left(1+2 \times\left(\frac{2 \mathrm{eV}}{L^{2}}\right)^{1 / 2} \frac{\delta t}{M_{2}^{1 / 2}}\right)
\end{array}\right.
$$

Plotting the reduced mass $\left(\mu_{1}=M_{1}^{D} / M_{1}\right)$ as a function of $\left(\mu_{2}=M_{2}^{D} / M_{2}\right)$ gives a linear equation of slope $\left(M_{1} / M_{2}\right)^{1 / 2}$. By using a pair of atoms in multi-hit events with significant difference in mass, Saxey's representation is, therefore, a good method to dissociate time and energy effects.

\section{Geometric Modeling of the Electrostatic Environment}

Currently, most of the ion trajectories studies are done by using analytical algorithms. Those are extremely complex to set up and are limited in precision because of some simplifications, such as the electrostatic geometry of the AP or the ion properties.

In our study, we use a commercial particle trajectory analysis software LORENTZ-2E V10.2 to model a straight flight path LEAP 5000 (CAMECA LEAP 5000 Atom Probe, 2020). The LEAP is modeled as an X-axisymmetric structure. The tip is modeled as a cone with a semi-circular apex of $R=60 \mathrm{~nm}$ and an angle of $82^{\circ}$ and biased at $V_{\mathrm{DC}}$. The tip-to-detector distance is fixed at $10 \mathrm{~cm}$. The $\mathrm{CE}$ is placed at $40 \mu \mathrm{m}$ off the tip and has an aperture of $40 \mu \mathrm{m}$. The CE (or the tip) was biased with different negative (positive for the tip) double exponential voltage pulses from 0.5 to $2 \mathrm{kV}$. A constant voltage was added on the tip to obtain a field of $30 \mathrm{~V} / \mathrm{nm}$. The MCP detector of $35 \mathrm{~mm}$ of radius and the shield are grounded. We chose a field solver type operating in a quasitransient mode to be able to investigate the temporal effect of the VP. By using the boundary element method (BEM), we discretize all the surfaces to solve the field evaporation. Then, we define emitter characteristics (mass, charge, $x, y$, departure time) and use a fifth-order adaptive step Runge-Kutta (RK5) algorithm to compute trajectories from 1,000 departure times of the VP. Each sample of the pulse corresponds to an ion evaporate at a precise time of departure on pulse. For each point, ionization probability and TOF can be calculated for the different elements $\left(\mathrm{H}^{+}, \mathrm{B}^{2+}, \mathrm{B}^{+}, \mathrm{Fe}^{2}\right.$ 


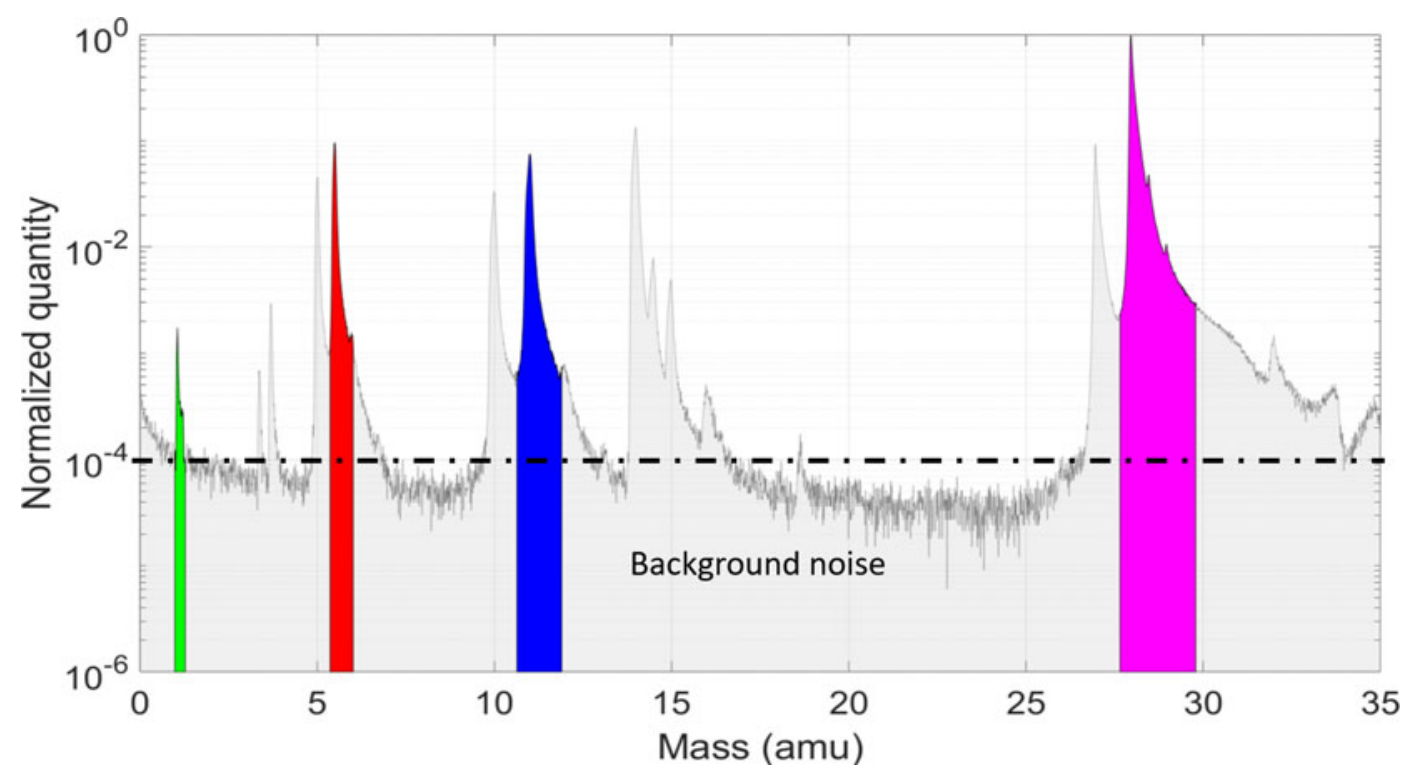

Fig. 4. Mass spectrum of FeBSi alloy. Mass peak of $\mathrm{Fe}^{2+}=28 \mathrm{amu}$ (magenta), $\mathrm{B}^{+}=11 \mathrm{amu}$ (blue), $\mathrm{B}^{2+}=5.5$ amu (red), and residual $\mathrm{H}^{+}=1$ amu (green).

$\left.{ }^{+}\right)$. Note that systematic errors in flight length $L$ are corrected by post processing and are so neglected in our study.

\section{Experimental Results}

\section{Mass Spectrum Resolution}

Experimental mass spectrum (Fig. 4) results show that the mass peaks resolution varies as a function of the corresponding ion mass. We determine the mass resolution of the peaks: $\mathrm{Fe}^{2+}, \mathrm{B}^{+}$, $\mathrm{B}^{2+}$, and the residual $\mathrm{H}^{+}$.

The mass resolution $M / \Delta M_{x \%}$ defines the quality of the mass spectrum, with $\Delta M$ the width of the mass peaks at $x \%$ of the maximum peak. Many factors influence the resolution: geometrical factors due to the geometry of the atom probe; accuracy of measurement due to the precision of the devices (Loi et al., 2013; Zhao et al., 2015; Pareige et al., 2016); and physical factors: temperature and energy deficit (Vurpillot, 2016). The following table shows the mass resolution of the different peaks of Figure 4.

Considering Table 1 where the mass resolution is determined for each peak of $\mathrm{Fe}^{2+}$ (magenta), $\mathrm{B}^{+}$(blue), $\mathrm{B}^{2+}$ (red), and residual $\mathrm{H}^{+}$(green), we observe the mass resolution increases proportionally to the square of the ion mass. Thus, it is easier to have a good quantification of $\mathrm{Fe}$ atoms than for lower mass, for example $\mathrm{H}$.

\section{Correlation Maps}

Correlation maps reflect the simultaneous evaporation of ions $i$ and $j$ from the specimen surface at some time, $\delta t$ after the pulse. Their times of flight are, therefore, each extended by $\delta t$, and they fall along a slightly curved track in the correlation map. It is clear from comparison with the conventional mass spectrum that these tracks, whose intensity falls off with distance from any coincidence points [pairs $\left(m_{1}, m_{2}\right)$ ], track shapes depending on energy/time spreads as indicated in equations (7) and (8) and correspond to the tails following each mass peak in mass spectra. In mass spectra, peak tails from different $m / n$ overlap with each other. However, the pair-wise correlation map
Table 1. Mass Resolution of the Mass Peaks: $\mathrm{Fe}^{2+}$ (Magenta), $\mathrm{B}^{+}$(Blue), $\mathrm{B}^{2+}$ (Red), and Residual $\mathrm{H}^{+}$(Green) (Fig. 4).

\begin{tabular}{lllll}
\hline Peaks & $\mathrm{H}^{+}$ & $\mathrm{B}^{2+}$ & $\mathrm{B}^{+}$ & $\mathrm{Fe}^{2+}$ \\
\hline Resolution $50 \%$ & 30 & 80 & 85 & 400 \\
\hline Resolution $10 \%$ & 5 & 30 & 40 & 95 \\
\hline Resolution $1 \%$ & NA & 8 & 10 & 30 \\
\hline
\end{tabular}

separates these tails in two dimensions and provides a clearer picture of the processes underlying the spectrum noise. Three correlation maps generated from the field evaporation of a bulk-metallic glass sample under different conditions are presented in Figure 5. They illustrate the understanding that may be gained from this approach. The data were obtained in voltage pulsing mode, at a pulse fraction of $20 \%$, an ion detection rate of $5 \times 10^{-3}$ atom/pulse, and a temperature of $50 \mathrm{~K}$. Although only weak diagonal trails are observed following each peak in the histogram, they extend throughout the entire pulse repetition timeperiod, in this case $5 \mu \mathrm{s}$, and into the following pulse window. These tails are responsible for the significant background noise observed in the mass spectrum.

Tracks that can be observed in VP mode are interestingly different compared with conventional dissociation tracks observed in laser-assisted evaporation of semiconductors or oxides compounds. As displayed in Figure 5 , the tracks of $\left(\mathrm{B}^{2+}, \mathrm{B}^{+}\right)$have a comma shape. It is impossible from this shape to interpret tails as effects of simple energetic errors or time dispersion. Indeed, the comma tracks have components different from a voltage dispersion [equation (8)] or from a temporal dispersion [equation (9)]. Conversely, the shape indicates a complex interplay between temporal and energy spreads.

This innovative approach in voltage AP has shown some uncommon "comma", which could be representative of the dynamic effects on correlated event due to the electrical field variation around the surface of the surface. Note that the higher is the difference of mass between the ion pair, the larger is the comma. 


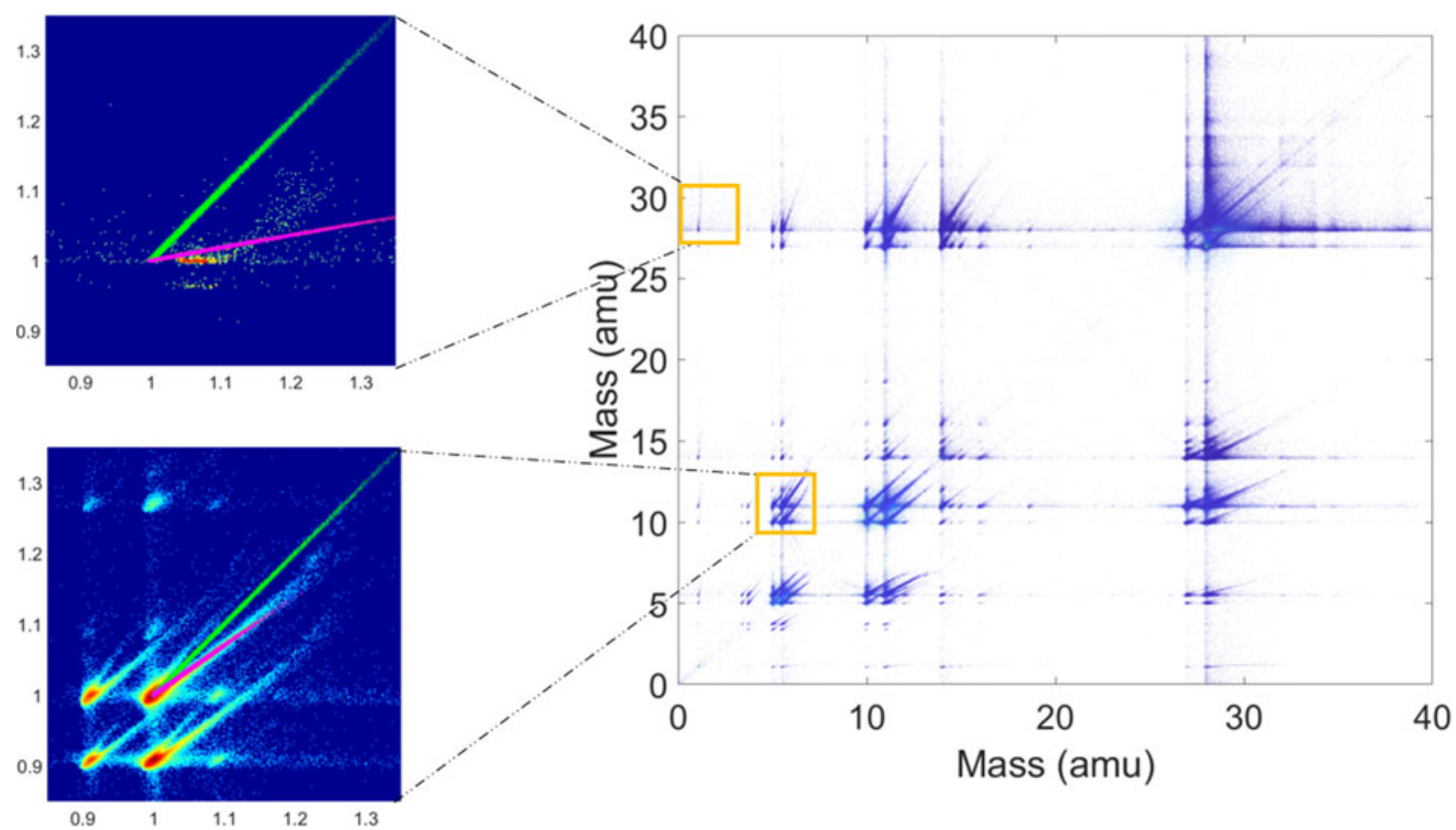

Fig. 5. Right: correlation map of FeBSi corresponding to the mass spectrum of Figure 4. The data were obtained in voltage pulsed mode with a pulse fraction of $20 \%$ of VDC. The density of points represents the density of correlated event. Note that boron is principally evaporated in $\mathrm{B}^{+} / \mathrm{B}^{2+}$ coevaporation. Left: Normalized close-up view of the correlated histogram. It represents $\mathrm{H}^{+} / \mathrm{Fe}^{2+}$ (up) and $\mathrm{B}^{+} / \mathrm{B}^{2+}$ (down) coevaporation events. The color scale represents the logarithm of the number of counts after normalization. The green line represents the $\Delta V / V$ variation relative to equation (8) (slope 1 ) -the magenta line represents the $\Delta T / T$ variation relative to equation (9) (slope $\sqrt{m_{1} / m_{2}}$ ).

\section{Simulation Results and Discussion}

\section{Kinetic Energy}

In DC mode, ions are gradually accelerated from the tip to the detector. Most of the acceleration takes place in the first millimeter of flight due to the diverging nature of the generated field evaporation. This phenomenon is accentuated by the presence of the CE that shields the field evaporation after ions pass through the aperture. As a result, the ion trajectory can be approximately separated into two parts; the first part is the accelerated zone from the tip to the $\mathrm{CE}$, then the free flight zone from the $\mathrm{CE}$ to the detector. With standard voltage applied to the sample, ions cross the acceleration zone in less than a fraction of a nanosecond. In pulse mode, with a pulse applied on the $\mathrm{CE}$, a remnant negative electrical field causes a short deceleration of the ions. Considering the electrostatic geometry of the LEAP, a significant field evaporation gradient induced by the applied voltage on the electrode exists in a $2-3 \mathrm{~mm}$ zone after the CE aperture. As a result, deceleration occurs in the $2-10 \mathrm{~ns}$ duration after the ion starts. This duration is approximately the total pulse duration. Looking at the time spent by ions in the acceleration/deceleration zone, it is clear that deceleration effects in this configuration induce most of the dynamic effects (Fig. 6).

To understand the relationship between the mass of the emitted ion and the final acquired kinetic energy in VP mode, we evaluated the actual kinetic energy of an ion as a function of the departure time over the pulse. We compared this energy to the potential energy, which is also the kinetic energy that should be acquired by an ion in a DC case, with a negative continuous voltage applied to the CE. A $20 \%$ pulse fraction was used in this study (Fig. 7), and four species were involved $\left(\mathrm{H}^{+}, \mathrm{B}^{2+}, \mathrm{B}^{+}\right.$, and $\left.\mathrm{Fe}^{2+}\right)$. As expected, kinetic energy acquired by $\mathrm{Fe}^{2+}$ is close to the potential energy, which shows that CE removes most of dynamic effects for Fe. However, for low mass element, first, the maximal kinetic energy is much lower than the expected one, and second, the peak maximum is not centered with the VP maximum.

In AP, most of the ions are emitted close to the voltage peak maximum (at $t=0 \mathrm{~ns}$ ). Two consequences exist when calculating a mass spectrum from an experimental dataset. First, a systematic mass shift when applying equation (1) with a constant coefficient for all species will be observed. However, this mass shift can be easily corrected by using a mass-dependent correction factor. Second, ions are emitted on the top of the VP. Fifty percent of ions are emitted on a time window centered on this pulse maximum position with a width less than a nanosecond. This interval gives rise to an interval of acquired kinetic energy for low mass species much larger than the energy distribution predicted through equation (4).

\section{Influence of the Pulse Fraction on Mass Resolution}

The impact of the temporal effects on the mass spectrum calculation was studied by evaluating for different pulse fractions and the two mass extrema $\left(\mathrm{H}^{+}\right.$and $\left.\mathrm{Fe}^{2+}\right)$, the calculated masses as a function of the departure time (Fig. 8). As observed, dynamic effects significantly change the minimum mass that can be calculated whatever the initial mass. It is clearly observed when a negative pulse applied to the CE triggers the evaporation. Conversely, when applying the (positive) VP to the tip, we note no sensible dynamic effects. This reinforces the interpretation of dynamics effects as the result of deceleration induced by the presence of the remnant field just after the CE. When voltage is applied to 


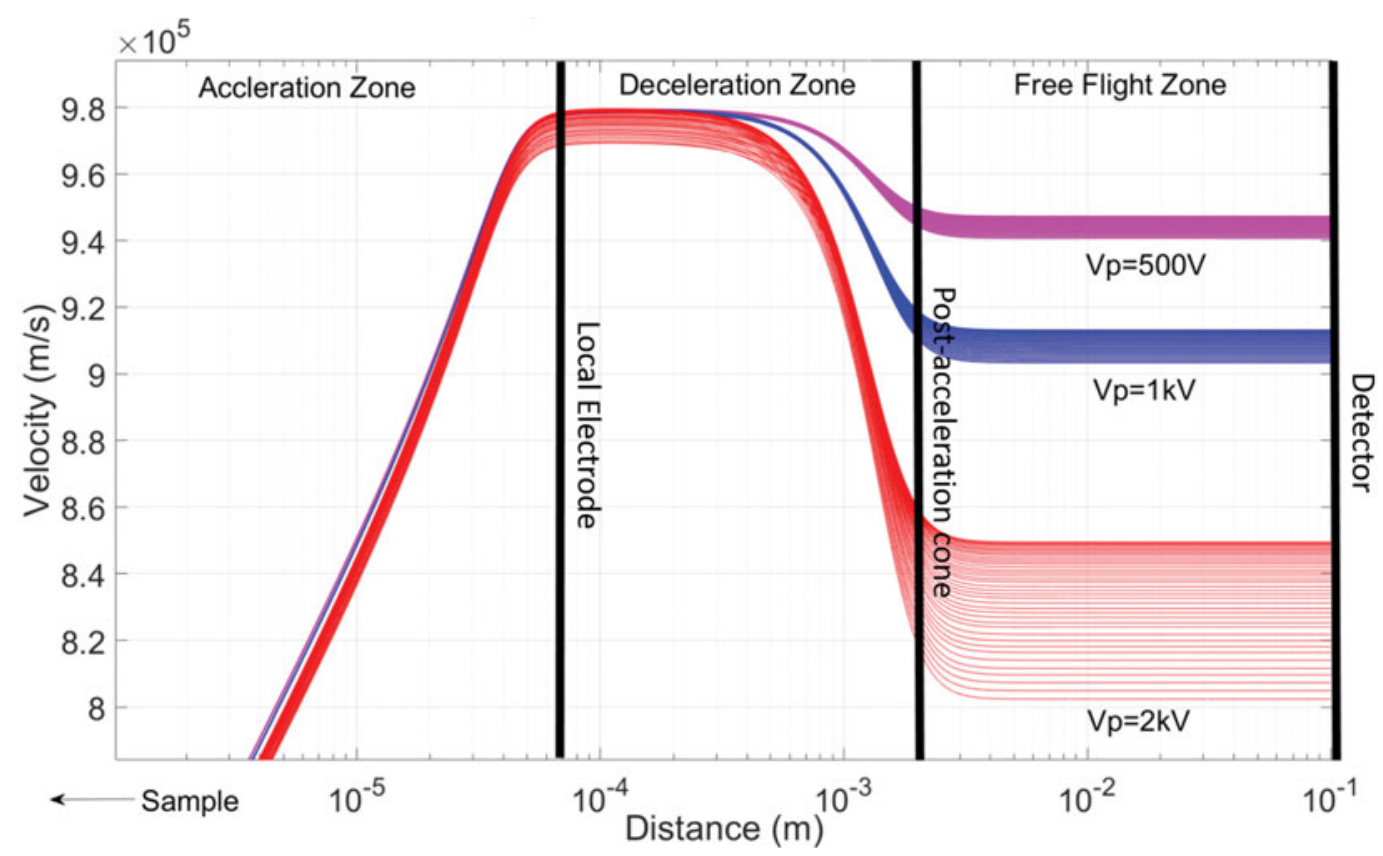

Fig. 6. Velocity as a function of the distance for ions $\mathrm{H}^{+}$for different VP amplitude [500 V (magenta)-1 kV (blue)-2 kV (red)] applied on the CE. Each line corresponds to an evaporated ion, which has left the sample at a precise departure time corresponding to a defined potential energy on the pulse. Note that increasing the amplitude amplifies the dynamic effects by amplifying the deceleration due to the negative field after the CE.

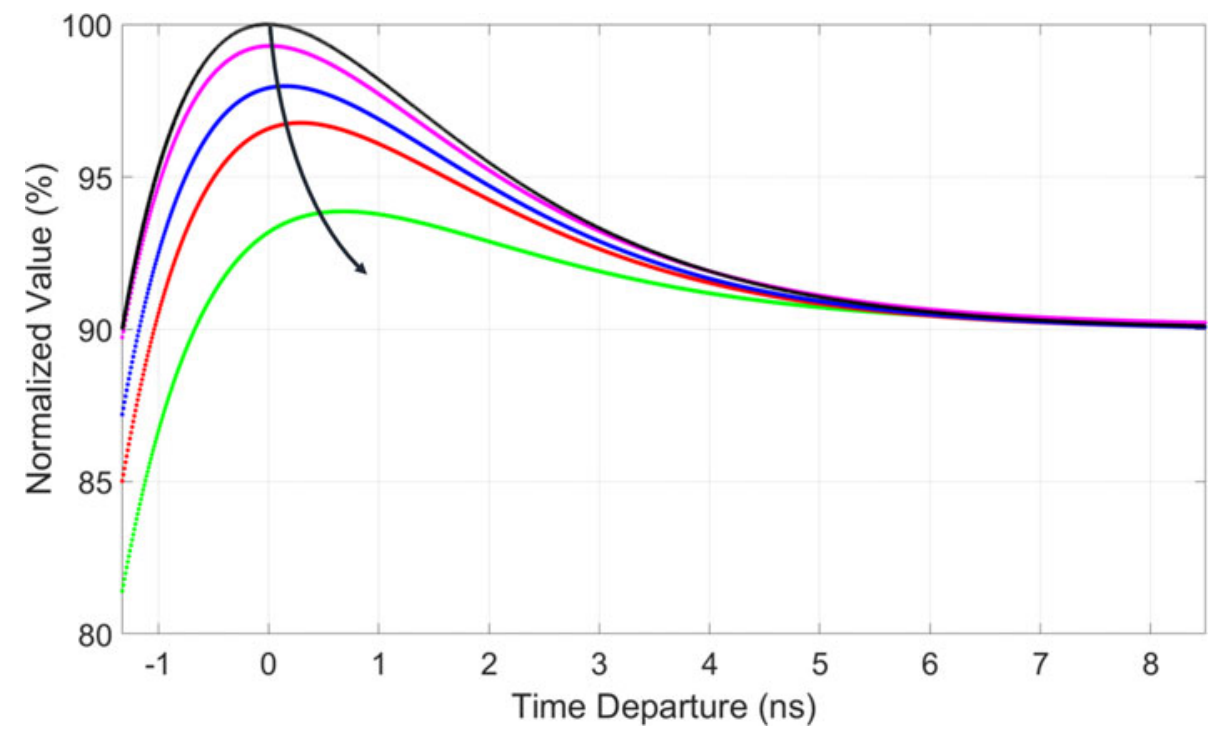

Fig. 7. Kinetic energy of $\mathrm{Fe}^{2+}$ (magenta), $\mathrm{B}^{+}$(blue), $\mathrm{B}^{2+}$ (red), and $\mathrm{H}^{+}$(green) compared with the potential energy (black) for a pulse fraction of $20 \%$. The calculations are realized following the ion trajectories simulation on Lorentz-2E. Energy deficit can be interpreted as the ratio between the reference and the ion kinetic energy. This deficit is higher for light ions (e.g., $\mathrm{H}^{+}$) and more important during the first part of the maximum of the pulse. The dynamic effects introduce a shift of the minimum of deficit energy, which is stronger for lower ion mass.

the tip, the $\mathrm{CE}$ acts as a grounded shield. We note also that dynamic effects are stronger for large pulse fraction, even if the time spread (width of the evaporating pulse) is smaller.

The exact impact of dynamic effects are calculating by determining a mass spectrum shape from these calculated curves. We may note that building a theoretical mass spectrum is not trivial. This is illustrated in Figure 9. There are several departure times that give the same mass over charge calculated ratio. Since for each departure time, there is a given probability of evaporation. A mass spectrum must be evaluated by adding these quantities. To represent the mass spectrum of these variations, we employ the following methodology.

If we consider a given VP, for each departure time $t_{\mathrm{D}}$, we can estimate a calculated mass $M$ using equation (1), and a probability of evaporation $P$. This gives us two curves $M=f\left(t_{\mathrm{D}}\right)$, and $P=f\left(t_{\mathrm{D}}\right)$ as schematically displayed in Figure 9. For an interval of mass $(M$, $\delta M)$, several intersection zones exist $\left(T_{\mathrm{D} i}, \delta t_{i}\right)_{\{i=1, \mathrm{~N}\}}$ and we need to add the total quantity of atoms evaporating at different times of 
(a)

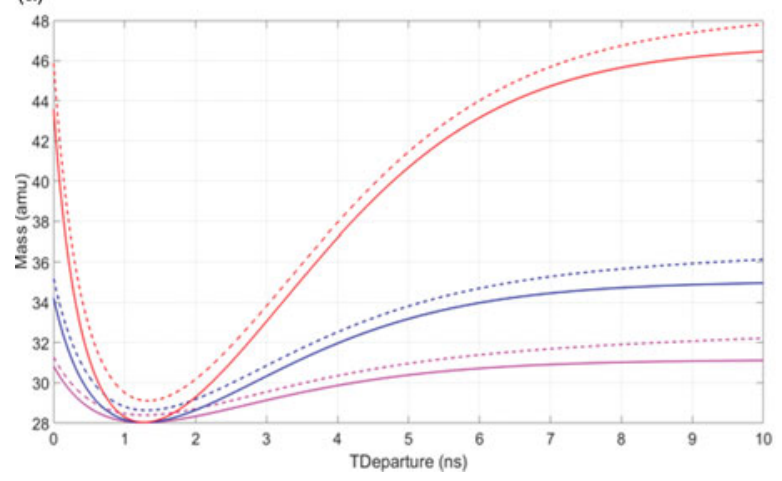

(b)

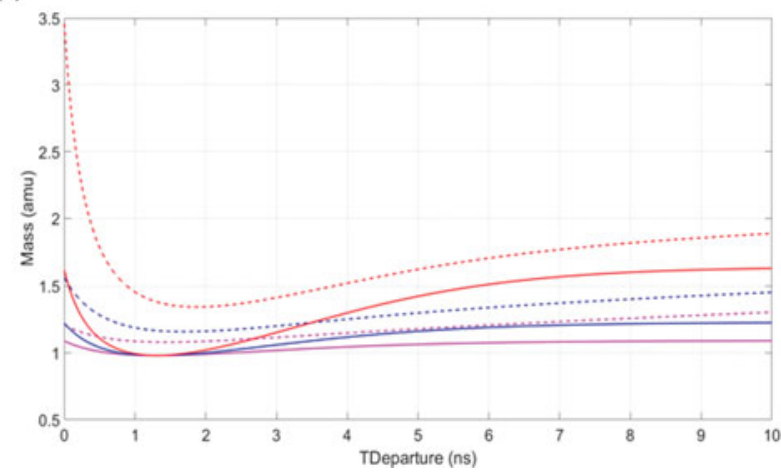

Fig. 8. (a) Mass variation of $\mathrm{Fe}^{2+}$ as a function of the departure time. (b) Mass variation of $\mathrm{H}^{+}$as a function of the departure time. Dashed lines: pulse on $\mathrm{CE} V_{\mathrm{p}}<0$, solid lines: pulse on sample $V_{\mathrm{p}}>0$, red: $2 \mathrm{kV}-\alpha_{\mathrm{p}}=40 \%$, blue: $1 \mathrm{kV}-\alpha_{\mathrm{p}}=20 \%$, magenta: $500 \mathrm{~V}-\alpha_{\mathrm{p}}=10 \%$.

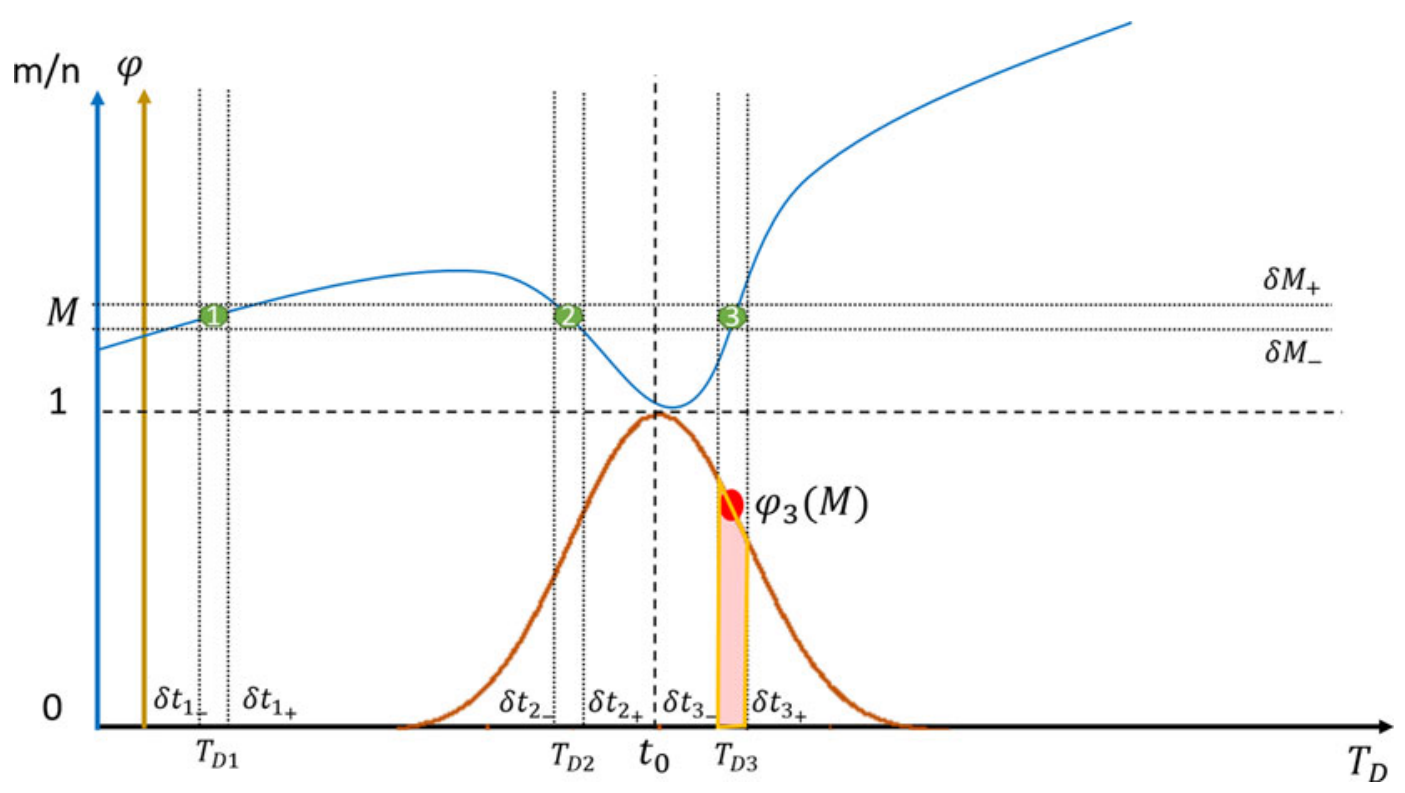

Fig. 9. Schematic graphic to represent equation (10). Blue line represents the normalized value of the mass-to-charge ratio as a function of the departure time. Red line represents the corresponding field evaporation pulse following the Arrhenius law [Equation (3)] calculated for each VP.

the VP. For each intersection zones, we need to estimate the width $\delta t_{i}$ to evaluate the actual amount $N_{i}(M)$ of ions that adds to the mass spectrum. In a first approach, the relationship between the mass bin of the mass spectrum $\delta M$ and $\delta t_{i}$ is straightforward, since $\delta M \sim\left(\mathrm{d} M / \mathrm{d} t_{i}\right) \delta t_{i}$. The derivative $(\mathrm{d} M / \mathrm{d} t)_{i}$ is numerically calculated using the calculated $\left(M, T_{\mathrm{D}}\right)$ list. The calculated atom count on each mass interval is then given by

$$
N(M)=\sum_{i}^{n} \varphi_{i}(M) \mathrm{d} t_{i}(M)=\sum_{i}^{n} \frac{\varphi_{i}(M)}{(\mathrm{d} M / \mathrm{d} t)_{i}} \Delta M
$$

We may note that this procedure neglects the time dispersion induced by time measurement errors, which limits the mass resolution to about 200 FWHM for $\mathrm{H}^{+}$and 1,000 FWHM for $\mathrm{Fe}^{2+}$. The result of the methodology is presented in Figure 10 for $\mathrm{H}^{+}$ and $\mathrm{Fe}^{2+}$, for different pulse fractions (from 10 to $40 \%$ ), pulsing on the tip or on the CE. As observed through this calculation, the shape of the mass peaks remains similar for $\mathrm{Fe}^{2+}$, whatever the pulsing mode, or the pulse fraction, which explains the experimental good mass resolution. For $\mathrm{H}^{+}$, the shape of peaks is strongly enlarged when high pulse fraction is used, and when pulses are applied on the CE. Conversely to this effect, applying the pulse directly to the tip should dramatically improve the mass resolution in this case.

When the same pulses $\left(\alpha_{\mathrm{p}}=10-40 \%\right)$ are applied positively on the sample, it strongly reduces the energy deficit and all peaks are centered on the same mass.

\section{Saxey Analysis}

Correlated events correspond to a couple of ions leaving at the same time from the surface of the tip apex. Following our trajectory simulations on Lorentz-2E, we can plot the detected ion mass-to-charge as a function of the departure time from the pulse (Fig. 11). To obtain the Saxey diagram, we simply plot the detected ion mass-to-charge in relation to each other. The size of the spots is determined as a function of the electrical 

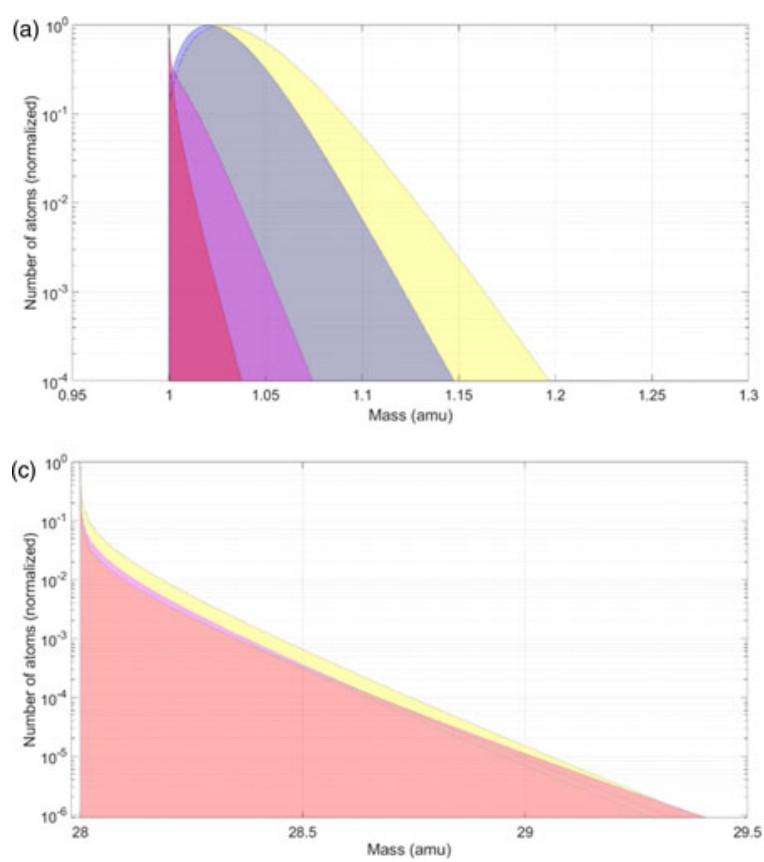
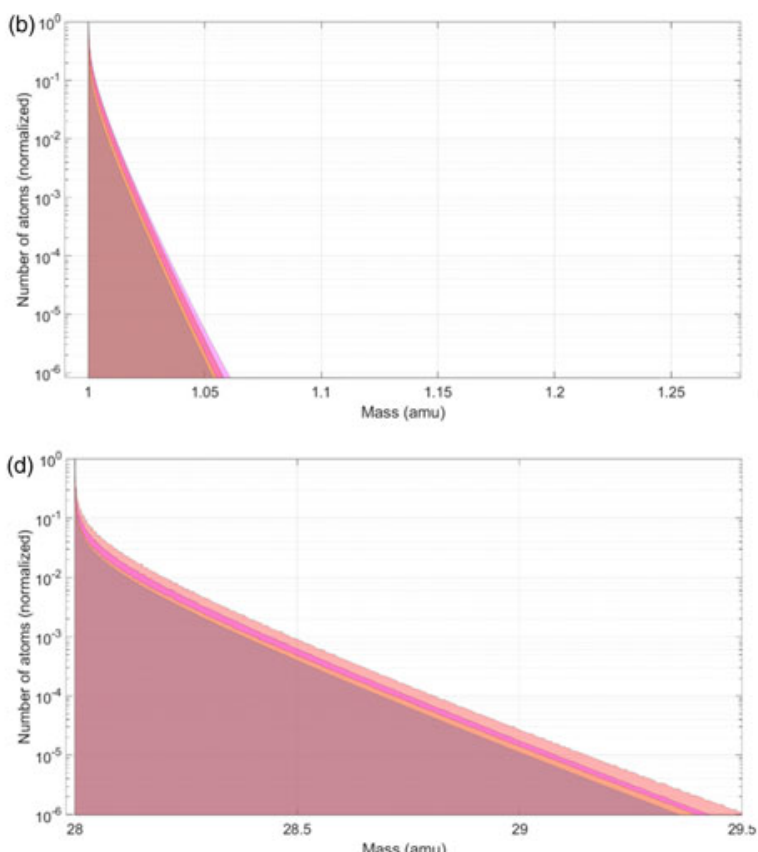

Fig. 10. Mass spectra of $(\mathbf{a}) \mathrm{H}^{+}$and $(\mathbf{c}) \mathrm{Fe}^{2+}$ with a pulse applied on $\mathrm{CE}\left(V_{\mathrm{p}}<0\right)$. Mass spectra of $(\mathbf{b}) \mathrm{H}^{+}$and $(\mathbf{d}) \mathrm{Fe}^{2+}$ with a pulse applied on sample $V_{\mathrm{p}}>0$. Red: $500 \mathrm{~V}$ $-\alpha_{\mathrm{p}}=10 \%$, magenta: $1 \mathrm{kV}-\alpha_{\mathrm{p}}=20 \%$, blue: $1,500 \mathrm{~V}-\alpha_{\mathrm{p}}=30 \%$, and yellow: $2,000 \mathrm{~V}-\alpha_{\mathrm{p}}=40 \%$.
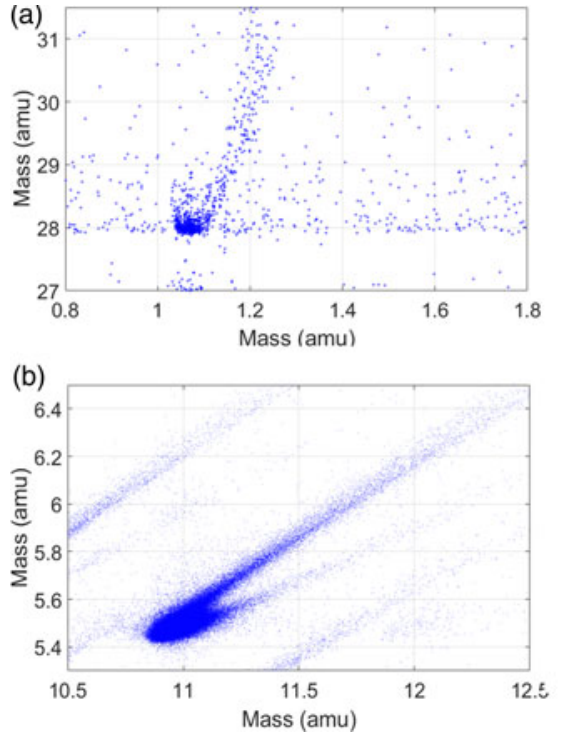

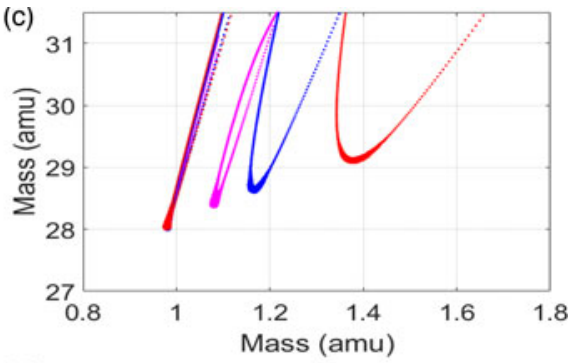

(d)

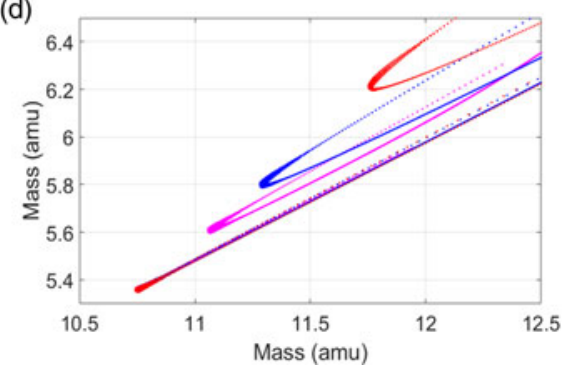

Fig. 11. Saxey diagram of $\mathrm{H}^{+} / \mathrm{Fe}^{2+}$ : (a) experimental data from an FeBSi alloy and (b) simulated results from Lorentz-2E and Saxey diagram of $\mathrm{B}^{+} / \mathrm{B}^{2+}$ : (c) experimental data from an FeBSi alloy and $(\mathbf{d})$ simulated results from Lorentz-2E of $\mathrm{B}^{+} / \mathrm{B}^{2}+$. The VP is applied on $\mathrm{CE}\left(V_{\mathrm{p}}<0\right)$ or on sample $\left(V_{\mathrm{p}}>0\right)$. Red: $2 \mathrm{kV}-\alpha_{\mathrm{p}}=$ $40 \%$; blue: $1 \mathrm{kV}-\alpha_{\mathrm{p}}=20 \%$; magenta: $500 \mathrm{~V}-\alpha_{\mathrm{p}}=10 \%$. Experimental data are corrected in mass on IVAS software and have a good correlation with simulated results when a pulse fraction of $\alpha_{\mathrm{p}}=20 \%$ is applied on the CE.

field. The bigger the spot, the higher is the probability of ionization.

Figure 11 demonstrates a good correlation with experimental results. As explained in the previous paragraph, the comma shape is strongly dependent of the ion mass difference, the VP fraction, and the pulse configuration. When the pulse is applied on the $\mathrm{CE}$, the conservation law is not applicable. Indeed, if we assume that the acceleration is instantaneous, the couple of ions would see the same energy deficit $\delta E$ as demonstrate in equation (7). If two ions leave at the same instantaneous moment from the tip surface (correlated field evaporation), they should have the same energy deficit $\delta E$ as explained in equation (8). However, this relationship is not true considering dynamic effects. The faster ion (lighter ion) will acquire less energy than the slower ion (heavier ion). The strong visual agreement between the model and the experimental Saxey analysis indicates that dynamic effects are the main causes of deterioration of the mass spectrum for light ions and must be taken into account when interpreting APT data.

When the pulse is applied on the sample (and the CE is grounded), the residual field after the CE can be neglected; 
there is no deceleration region. Moreover, the acceleration can be considered as instantaneous because the field evaporation directly drops in the few nanometers in front of the sample. Therefore, when the couple of ions leave the surface, they do not see the pulse variation, which is responsible of the dynamic effects. Only the time dispersion and the initial potential energy are the issues. Equation (8) can be considered as true, and that explains the straight line visible for different VP fraction.

\section{Dynamic Effects Consequences on Chromatic Aberrations}

In most of the classical methods of reconstruction, algorithms have been designed based upon some assumptions: field expressions around the sample are represented by a hyperboloidal or a paraboloidal model due to the approximation tip apex represented by a spherical surface of radius $R$ on a truncated cone with a shank angle $a$. Within this assumption, the standard equation of the movement relative to the field distribution is written as (de Castilho, 1999):

$$
\begin{aligned}
2[\phi(x, z)-1] \frac{\mathrm{d} x^{2}}{\mathrm{~d} z^{2}}= & {\left[\frac{\mathrm{d} \phi(x, z)}{\mathrm{d} x}-\frac{\mathrm{d} \phi(x, z)}{\mathrm{d} z} \frac{\mathrm{d} x}{\mathrm{~d} z}\right] } \\
& \times\left(1+\left.\frac{|\mathrm{d} x|^{2}}{\mathrm{~d} z}\right|^{2}\right)
\end{aligned}
$$

With: $x=r \sin (\theta)$ and $z=r \cos (\theta)$ in polar, $\phi$ electrical field. From these equations, it is then possible to obtain the ion trajectories by numerical integration.

Then, the position of the surface of the sample $(x, y)$ are deduced from the ion's impact on the detector $(X, Y)$, thanks to a projection law (Southworth \& Walls, 1978), equation (12):

$$
\left\{\begin{array}{l}
x=\frac{X}{M}=\frac{X}{L} \xi R \\
y=\frac{Y}{M}=\frac{Y}{L} \xi R
\end{array}\right.
$$

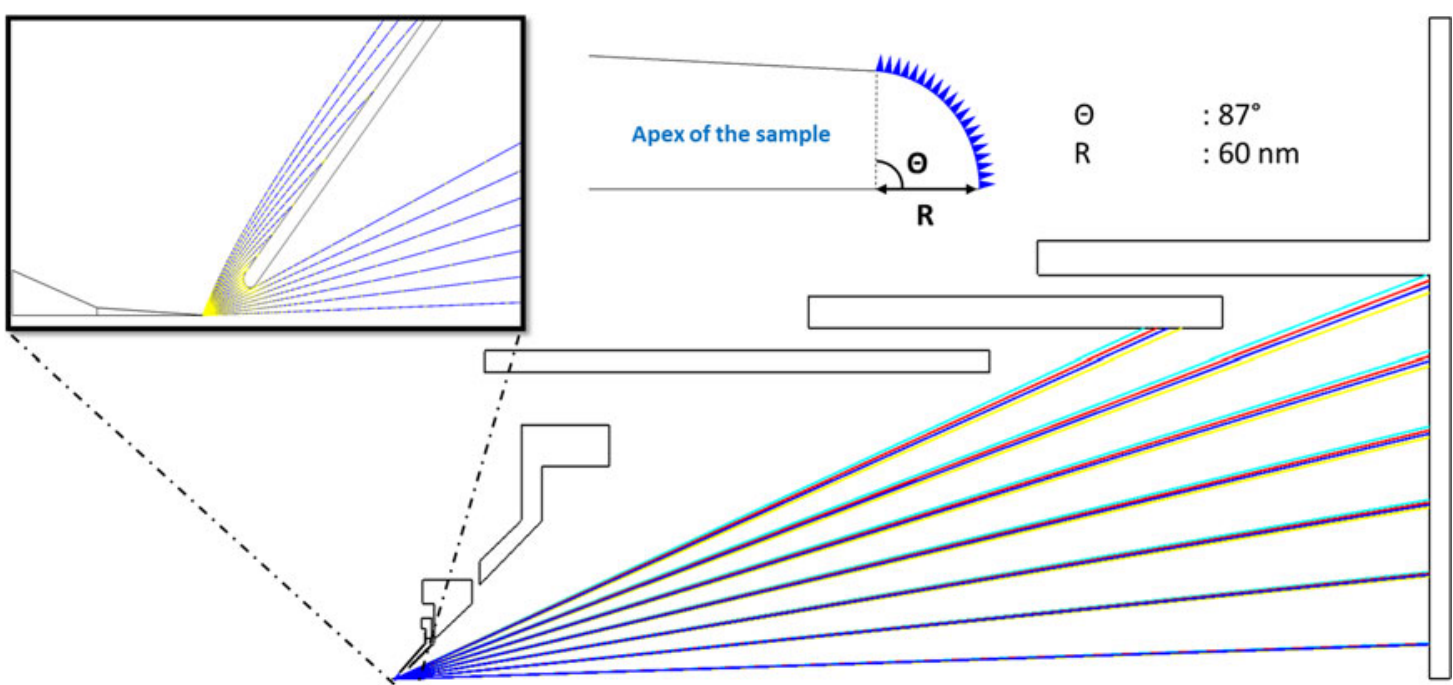

Fig. 12. Trajectory representations of ions: $\mathrm{Fe}^{2+}$ (yellow), $\mathrm{B}^{+}$(blue), $\mathrm{B}^{2+}$ (red), and $\mathrm{H}^{+}$(cyan) for a pulse fraction of $10 \%$ ( $1 \mathrm{kV}$ ). Close-up view of the trajectories between the sample and the local electrode. The curvature of the trajectory corresponds to the compression factor $\xi$. where $M$ is the magnification factor and $\xi$ the compression factor (between 1 and 2). In our study, we demonstrate that the trajectory of an ion is also relative to its own mass and the initial potential energy received. We will use the classical equation (12) to determine the variation of the compression factor $\xi$ as a function of the mass, and the VP fraction. For this simulation, we consider a pulse fraction of $20 \%\left(V_{\mathrm{p}}=1 \mathrm{kV}\right)$ applied on the $\mathrm{CE}$, and the ion is ionized at the maximum of $V_{\mathrm{p}}$. This pulse is responsible for a diverging trajectory close to the tip surface during the acceleration period, which will be strongly magnified on the detector (Gault et al., 2009). In this paragraph, we do not consider local atomic movements prior to ionization (e.g., surface diffusion and rolling-up) which could perturb both the electrostatic environment and ion trajectories [such as in Marquis \& Vurpillot (2008)]. The apex of the sample is hemispherical, and 20 starting positions on the apex are defined homogeneously. The detector field of view on our sample is equal to $52^{\circ}$.

Regarding to Figure 12, we can easily notice that trajectories are not identical for all ions starting at the same positions. For lighter ions (e.g., $\mathrm{H}^{+}$), the trajectory heads to the outside of the detector. Thus for two different ions leaving at the same time and the same position $(x, y)$ from the tip apex, the final $Y$ position on the detector will be different. If we take the final position for two extrema $\left(\mathrm{Fe}^{2+}\right.$ and $\left.\mathrm{H}^{+}\right)$, we can represent (Fig. 13) the impact difference as a function of the angle of departure. We can remark that this evolution is linear and increases with the launch angle $\theta$.

Second, we can plot the relative TOF errors, which occur for different flight paths and different mass-to-charge (Fig. 14). It corresponds to the well-known bowl function used to correct the TOF of ions depending the impact on the detector for mass calibration (Larson, 2013). This allows to correct spatially the mass resolution. As expected from the simulation, the bowl function is mass dependent.

According to the precise simulation of the ion trajectories, we can quantify for the first time, the main parameters using during the reconstruction process. These correspond to the $k$ factor representing the slope from the variation of the $Y_{\mathrm{D}}$ position as a function of the $\theta$ angle of departure of the ion. Following the 


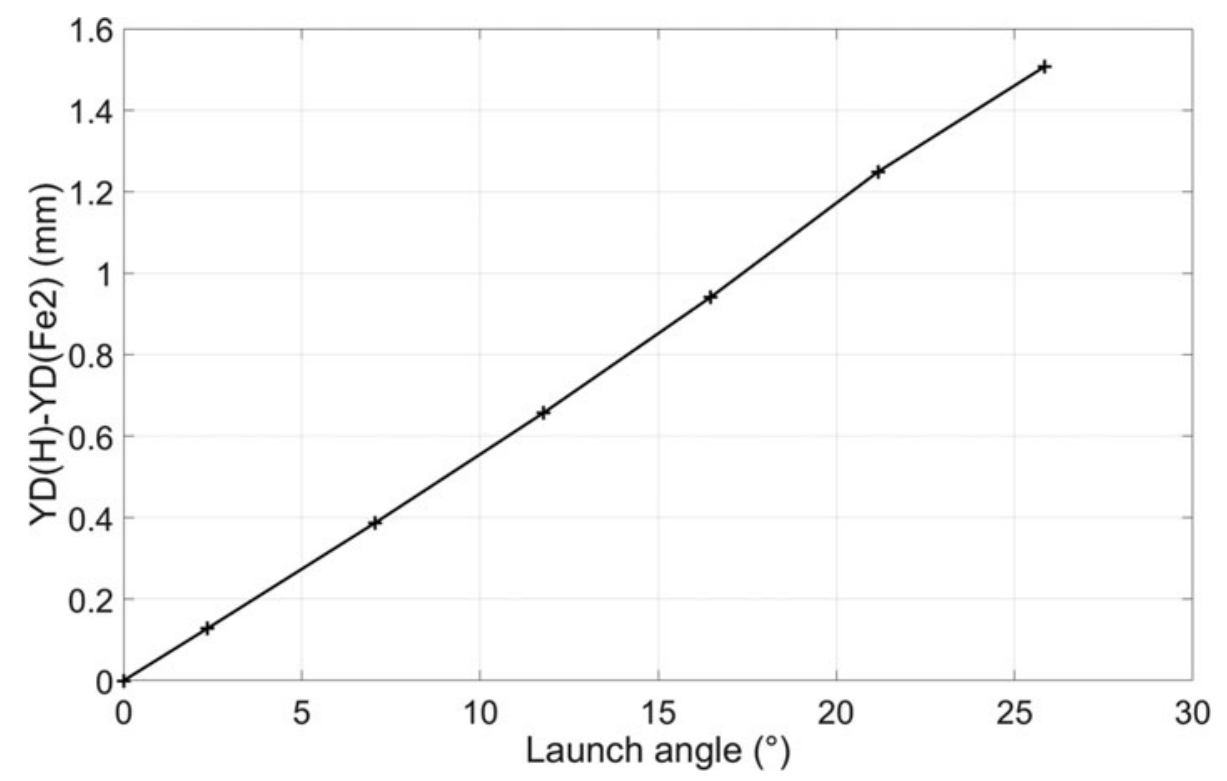

Fig. 13. Linear variation of $Y_{\text {detector }}$ difference between $\mathrm{H}^{+}$and $\mathrm{Fe}^{2+}$ as a function of the launch angle on the sample.

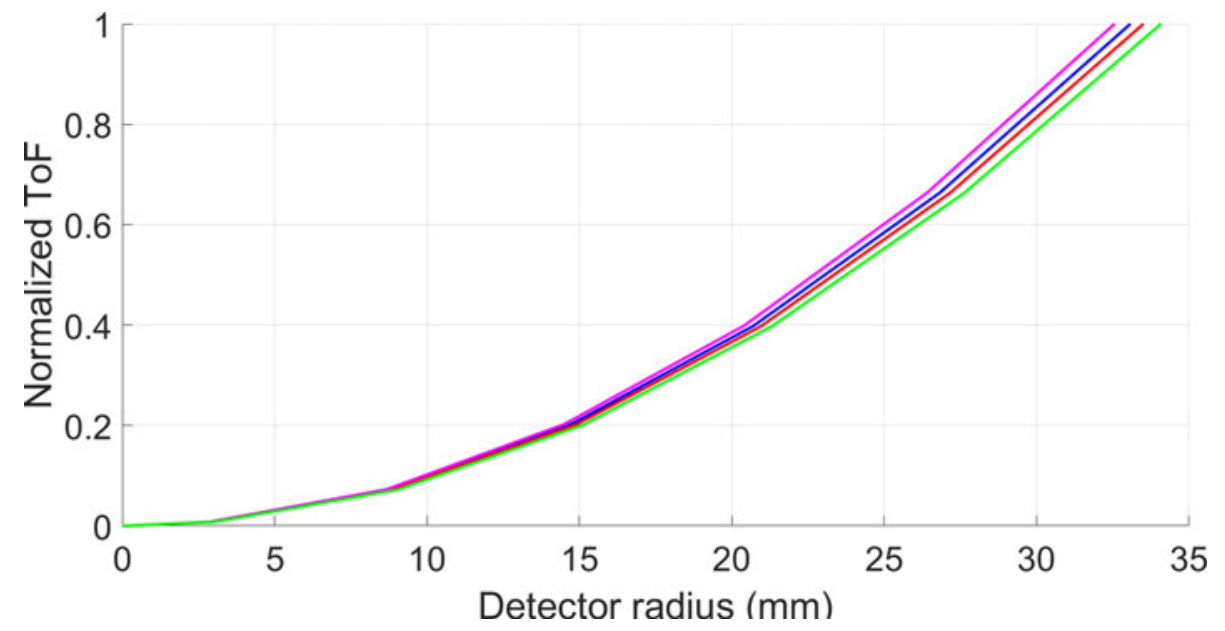

Fig. 14. Flight length errors and TOF isosurfaces. $\mathrm{Fe}^{2+}$ (magenta), $\mathrm{B}^{+}$(blue), $\mathrm{B}^{2+}\left(\right.$ red), and $\mathrm{H}^{+}$(green).

relation $\theta^{\prime}=\arctan \left(Y_{\mathrm{D}} / L\right)$, we can represent the factor of compression $\theta=\xi \theta^{\prime}$ which represents the curvature of the trajectory between the sample and the CE. By computing the $Y$ position on the detector relative to the $y$ position on the sample, we quantify the magnification factor $M$. These three parameters vary linearly as a function of the square of the ion mass and are resumed in Table 2:

Table 2. Reconstruction Parameter Quantification as Function of the Ion Mass.

\begin{tabular}{lcccc}
\hline Ions & Mass (amu) & $k \times 10^{-3}$ & $\xi$ & $M \times 10^{6}$ \\
\hline $\mathrm{Fe}^{2+}$ & 28 & 1.2566 & 1.4235 & 1.2290 \\
\hline $\mathrm{B}^{+}$ & 11 & 1.2764 & 1.4115 & 1.2480 \\
\hline $\mathrm{B}^{2+}$ & 5.5 & 1.2929 & 1.3947 & 1.2644 \\
\hline $\mathrm{H}^{+}$ & 1 & 1.315 & 1.3728 & 1.2861 \\
\hline
\end{tabular}

To give an example, the following graph (Fig. 15) represents the variation of the $Y_{\text {detector }}$ as a function of the ion mass. We can remark that at $V_{\mathrm{DC}}$ (no field variation), there is no trajectory differences between the ion mass. The $Y_{\text {detector }}$ impact is the same for all the ions (black curve). When a pulse is applied on the CE, there is a field variation responsible for chromatic aberrations. The lighter ions are more influenced and projected to the external side of the detector. The aberration is amplified when light ions leave from the wide angle of departure (blue curve).

To conclude, the chromatic aberration can be represented as a simple schematic (Fig. 16) of a detector with the impacts of a precipitate composed of two elements with a big mass difference, e.g., $\mathrm{Fe}^{2+} / \mathrm{H}^{+}$. The chromatic aberration appears as the difference of magnification of the two circles $\mathrm{Fe}^{2+}$ (magenta) and $\mathrm{H}^{+}$(green) and a transposition of the center of the circle from a DC ionization (solid line) to a pulse evaporation (dashed lines). This will have an impact on the $3 \mathrm{D}$ reconstruction of the sample, especially for small precipitates. 


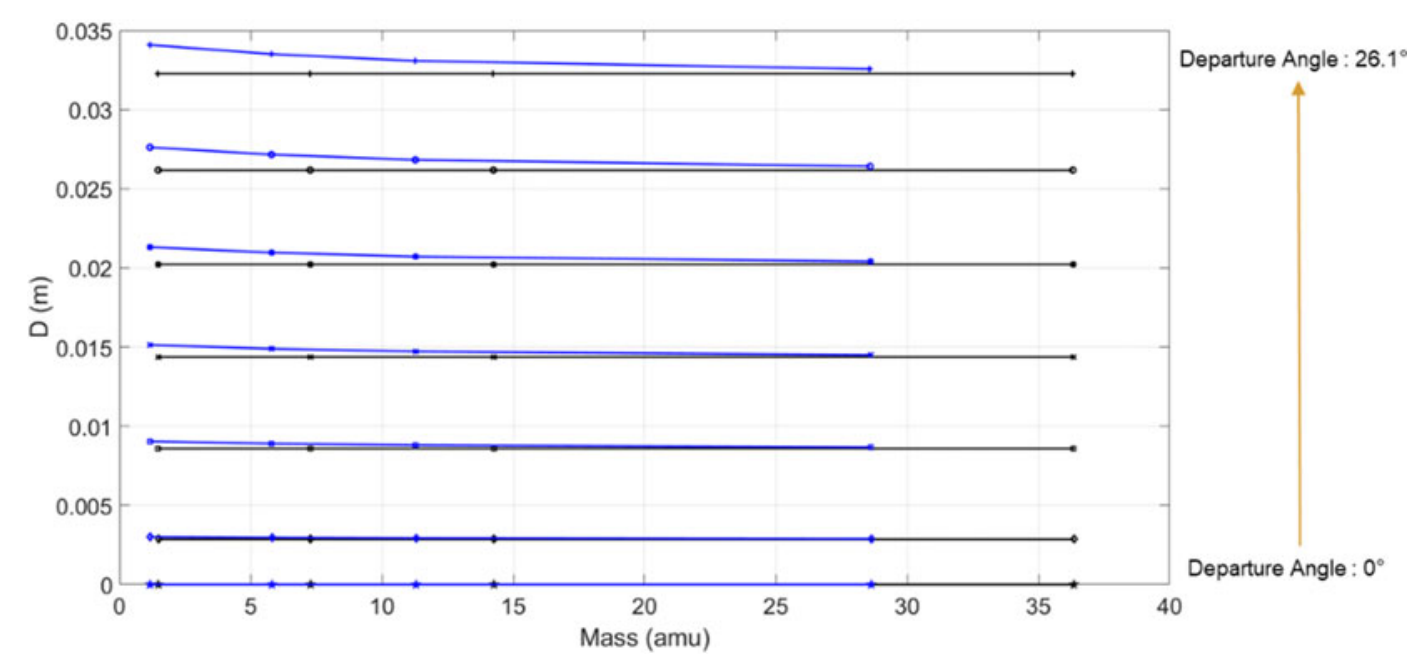

Fig. 15. $Y$ position on the detector as a function of the ion mass at $V_{D C}$ (black) and with a pulse of $1 \mathrm{kV}$ (blue).

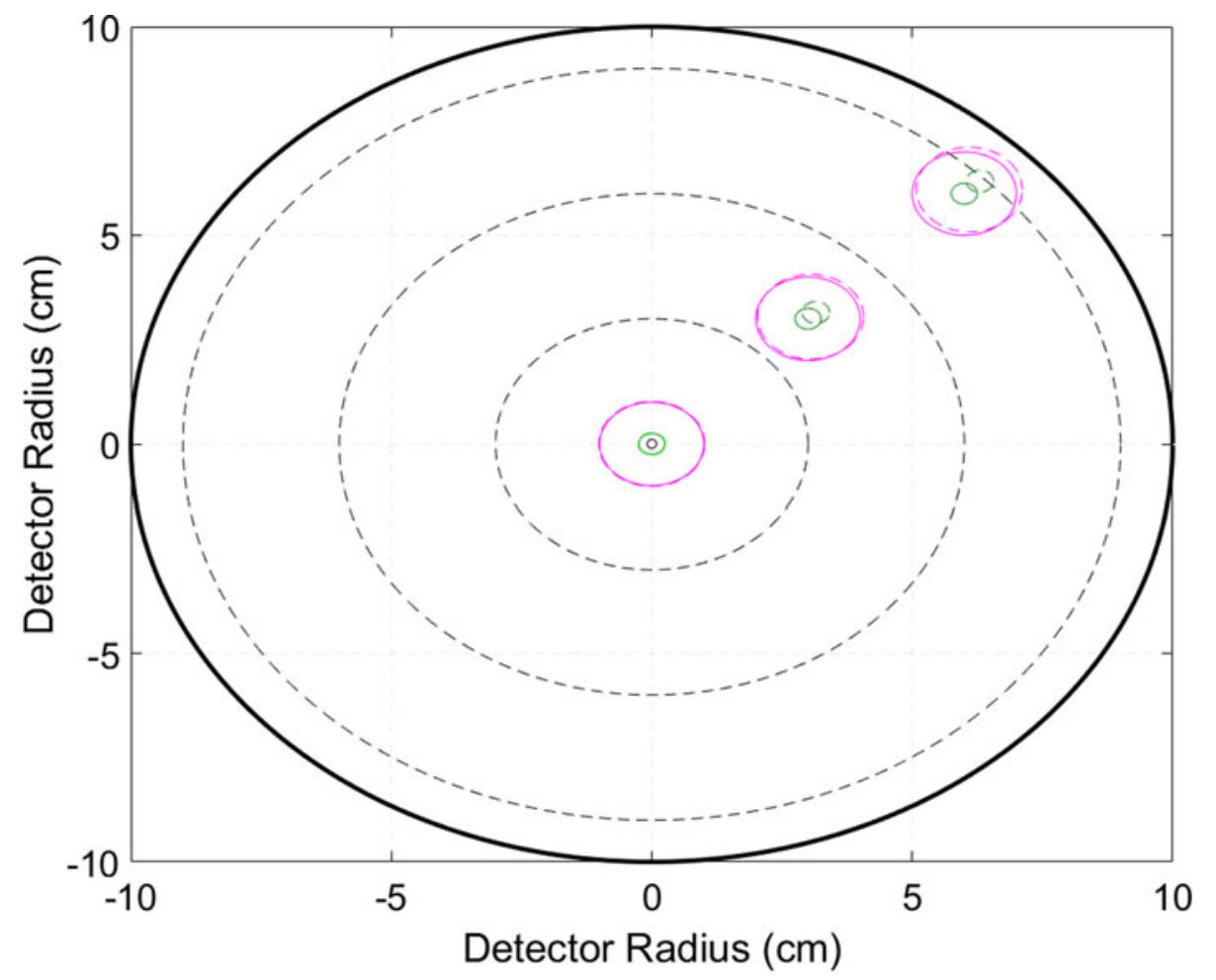

Fig. 16. Schematic representation of the chromatic aberration of a precipitate of $\mathrm{Fe}^{2+} / \mathrm{H}^{+}$(magenta/green) based on Table 2 results. The ideal positions of the precipitate are represented by solid line. The projections following the dynamic effects on trajectory are represented by the dashed lines. The chromatic aberration increase with the detector radius, and non-identically magnified the precipitate.

\section{Conclusion}

In this paper, we have investigated the influence of different highvoltage electrical pulses applied on the CE or on the specimen in a straight flight path LEAP 5000. By modeling the accurate electrostatic environment of the APT, we calculate different trajectories for a wide ion mass scale. This enables the investigation of light elements (e.g., $\mathrm{H}^{+}$) to medium elements (e.g., $\mathrm{Fe}^{2+}$ ).

We demonstrate that the energy deficit and the time dispersion are the main factor on the degradation of the mass resolution due to the field variation around the surface of the tip apex. The dependency with the voltage amplitude is strongly visible when the pulse is applied on the CE and for ion masses less than 10 amu. We also quantify these dynamic effects with an innovative approach, the Saxey diagram (Saxey, 2011). The correlation with experiments confirms our analysis.

In a second step, we have demonstrate that dynamic effects cause some trajectory deviations amplified by the pulse fraction, the angle of departure of the ions, and the ion mass. Again, light ions are sensitive to the pulse amplitude and the pulse waveform. However, by quantifying the variation of the compression factor as function of the mass, it is possible to correct the position of the detector impact. 
Dynamic effects can be reduced by applying an appropriate pulse directly on the specimen. Further studies are engaged to find a new pulse configuration for APT to optimize the mass resolution (Rousseau et al., 2020).

\section{Acknowledgments}

B.G. is grateful for the financial support of the Max-Planck Gesellschaft, Germany via the Laplace project. B.G. acknowledges financial support from the ERC-CoG-SHINE-771602, Germany. F.V. thanks also the financial support of the University of Rouen, France through a CRCT funding and the MaxPlanck-Institut für Eisenforschung $\mathrm{GmbH}$, Germany for financial support during his research stay.

\section{References}

Bémont E, Bostel A, Bouet M, Da Costa G, Chambreland S, Deconihout B \& Hono K (2003). Effects of incidence angles of ions on the mass resolution of an energy compensated 3D atom probe. Ultramicroscopy 95, 231-238. doi:10.1016/S0304-3991(02)00321-2

Breen AJ, Stephenson LT, Sun B, Li Y, Kasian O, Raabe D, Herbig M \& Gault B (2020). Solute hydrogen and deuterium observed at the near atomic scale in high-strength steel. Acta Mater 188, 108-120. doi:10.1016/ j.actamat.2020.02.004

CAMECA LEAP 5000 Atom Probe. (2020) Available at https://www.cameca. com/products/apt/leap-5000 (accessed January 27, 2020).

Cerezo A, Smith GDW \& Clifton PH (2006). Measurement of temperature rises in the femtosecond laser pulsed three-dimensional atom probe. Appl Phys Lett 88(15), 154103. doi:10.1063/1.2191412

Chen Y-S, Haley D, Gerstl SSA, London AJ, Sweeney F, Wepf RA, Rainforth WM, Bagot PAJ \& Moody MP (2017). Direct observation of individual hydrogen atoms at trapping sites in a ferritic steel. Science 355(6330), 1196-1199. doi:10.1126/science.aal2418

de Castilho CMC (1999). Ion trajectories in atom probe field ion microscopy and gas field ion sources. J Phys D: Appl Phys 32(17), 2261-2265. doi:10.1088/0022-3727/32/17/317

Deconihout B, Saint-Martin R, Jarnot C \& Bostel A (2003). Improvement of the mass resolution of the atom probe using a dual counter-electrode. Ultramicroscopy 95, 239-249. doi:10.1016/S0304-3991(02)00322-4

De Geuser F \& Gault B (2020). Metrology of small particles and solute clusters by atom probe tomography. Acta Mater 188, 406-415. doi: 10.1016/ j.actamat.2020.02.023

De Geuser F, Gault B, Bostel A \& Vurpillot F (2007). Correlated field evaporation as seen by atom probe tomography. Surf Sci 601(2), 536-543. doi:10.1016/j.susc.2006.10.019

EIKOS (2020). Available at https://www.cameca.com/products/apt/eikos (accessed January 27, 2020).

Gault B, Moody MP, de Geuser F, Haley D, Stephenson LT \& Ringer SP (2009). Origin of the spatial resolution in atom probe microscopy. Appl Phys Lett 95(3), 034103. doi:10.1063/1.3182351
Gault B, Moody MP, De Geuser F, La Fontaine A, Stephenson LT, Haley D \& Ringer SP (2010a). Spatial resolution in atom probe tomography. Microsc Microanal 16(1), 99-110. doi:10.1017/S1431927609991267

Gault B, Müller M, La Fontaine A, Moody MP, Shariq A, Cerezo A, Ringer SP \& Smith GDW (2010b). Influence of surface migration on the spatial resolution of pulsed laser atom probe tomography. J Appl Phys 108(4), 044904. doi:10.1063/1.3462399

Gruber M, Vurpillot F, Bostel A \& Deconihout B (2011). Field evaporation: A kinetic Monte Carlo approach on the influence of temperature. Surf Sci 605(23-24), 2025-2031. doi:10.1016/j.susc.2011.07.022

Kelly TF, Camus PP, Larson DJ, Holzman LM \& Bajikar SS (1996). On the many advantages of local-electrode atom probes. Ultramicroscopy 62(1-2), 29-42. doi:10.1016/0304-3991(95)00086-0

Larson DJ (2013). Local Electrode Atom Probe Tomography: A User's Guide. New York: Springer.

Larson DJ, Camus PP \& Kelly TF (1996). Optimal field pulsing for atom probes with counter electrodes. Appl Surf Sci 94-95, 434-441. doi:10.1016/0169-4332(95)00407-6

Loi ST, Gault B, Ringer SP, Larson DJ \& Geiser BP (2013). Electrostatic simulations of a local electrode atom probe: The dependence of tomographic reconstruction parameters on specimen and microscope geometry. Ultramicroscopy 132, 107-113. doi:10.1016/j.ultramic.2012.12.012

Marquis EA \& Vurpillot F (2008). Chromatic aberrations in the field evaporation behavior of small precipitates. Microsc Microanal 14(6), 561-570. doi:10.1017/S1431927608080793

Miller MK \& Forbes RG (2014). Atom-Probe Tomography. Boston, MA Springer US. doi:10.1007/978-1-4899-7430-3.

Müller EW (1951). Das Feldionenmikroskop. Z Phys 131(1), 136-142. doi:10.1007/BF01329651.

Pareige C, Lefebvre-Ulrikson W, Vurpillot F \& Sauvage X (2016). Chapter five-Time-of-flight mass spectrometry and composition measurements. In Atom Probe Tomography, Lefebvre-Ulrikson W, Vurpillot F \& Sauvage X (Eds.), pp. 123-154. Academic Press. doi:10.1016/B978-0-12-8046470.00005-X

Rousseau L, Normand A, Tehrani K \& Vurpillot F (2020). Characterization of a high voltage and high frequency pulse generator configuration for atom probe. In 2020 IEEE 15th International Conference of System of Systems Engineering (SoSE), pp. 193-198. Budapest, Hungary: IEEE. doi:10.1109/ SoSE50414.2020.9130486

Saxey DW (2011). Correlated ion analysis and the interpretation of atom probe mass spectra. Ultramicroscopy 111(6), 473-479. doi:10.1016/ j.ultramic.2010.11.021

Southworth HN \& Walls JM (1978). The projection geometry of the field-ion image. Surf Sci 75(1), 129-140. doi:10.1016/0039-6028(78)90057-2

Takahashi J, Kawakami K \& Kobayashi Y (2019). Application of atom probe tomography to fundamental issues of steel materials. Surf Interface Anal $\mathbf{5 1}$ (1), 12-16. doi:10.1002/sia.6535

Vurpillot F (2016). Chapter two-Field ion emission mechanisms. In Atom Probe Tomography, Lefebvre-Ulrikson W, Vurpillot F \& Sauvage X (Eds.), pp. 17-72. Academic Press. doi:10.1016/B978-0-12-804647-0.00002-4

Zhao L, Normand A, Delaroche F, Ravelo B \& Vurpillot F (2015). Pulse shaping optimization for improving atom probe tomography. Int J Mass Spectrom 386, 47-53. doi:10.1016/j.ijms.2015.06.012 